\title{
Levantamento florístico no cerrado de Pedregulho, SP, Brasil ${ }^{1}$
}

\author{
Denise Sasaki ${ }^{2,3}$ e Renato de Mello-Silva ${ }^{2}$
}

Recebido em 29/08/2006. Aceito em 24/05/2007

\begin{abstract}
RESUMO - (Levantamento florístico no cerrado de Pedregulho, SP, Brasil). Em Pedregulho, município do extremo nordeste do Estado de São Paulo, ocorrem fragmentos de cerrado considerados floristicamente distintos dos demais remanescentes paulistas. Nesse estudo, foi realizado um levantamento florístico em Pedregulho, abordando duas regiões geologicamente distintas: o Parque Estadual das Furnas do Bom Jesus e o distrito de Estreito. Em cada uma, delimitaram-se duas parcelas de $50 \times 50 \mathrm{~m}$, totalizando um hectare, onde foram encontradas 65 famílias e 379 espécies de angiospermas. Coletas em áreas adjacentes totalizaram 71 famílias e 443 espécies. As duas regiões estudadas têm baixa similaridade florística entre si. A distribuição geográfica das espécies é analisada e oito padrões são delimitados para aquelas cuja distribuição no Estado de São Paulo é restrita à região de Pedregulho.
\end{abstract}

Palavras-chave: Cerrado, levantamento florístico, Pedregulho, São Paulo

\begin{abstract}
Floristic inventory of cerrado at Pedregulho, São Paulo State, Brazil). Pedregulho municipality in the far northeastern region of São Paulo state has fragments of cerrado vegetation thought to be floristically distinct from other cerrado remmants in the state. In this study, a floristic survey was carried out at Pedregulho, focusing on two geologically distinct regions: Furnas do Bom Jesus State Park and the Estreito district. In each region, $50 \times 50 \mathrm{~m}$ plots were set up for a total sample area of 1 hectare, where 65 families and 379 angiosperm species were recorded. Collecting efforts in adjacent areas resulted in a total of 71 families and 443 species. The two study areas had low floristic similarity. Geographic species distribution was analyzed and eight patterns were defined for those species restricted to the Pedregulho region in São Paulo.
\end{abstract}

Key words: Cerrado, floristic inventory, Pedregulho, São Paulo

\section{Introdução}

O cerrado, com diferentes fisionomias, já cobriu cerca de 14\% do território paulista (Brito 1997; Durigan et al. 2004). Entre 1962 e 1992 houve uma redução aproximada de $87 \%$ desta área (Kronka et al. 1998) e, atualmente, há somente fragmentos isolados que correspondem a menos de $7 \%$ de sua área original (Durigan et al. 2003a). Esforços para conservar estes remanescentes resultaram em projetos para conhecer e divulgar sua biodiversidade, estabelecer áreas prioritárias e diretrizes para conservação, e promover a exploração econômica sustentável do cerrado (Brito 1997; Kronka et al. 1998; Durigan et al. 2002).

Os cerrados de São Paulo apresentam maior similaridade florística com áreas do sul de Minas Gerais e do Paraná, formando um grupo à parte dos cerrados das demais regiões de Minas Gerais e do Centro-Oeste (Ratter \& Dargie 1992; Ratter et al. 1996; Ratter et al.
2003). Dividem-se ainda em dois grandes grupos: um de áreas do centro para o nordeste-norte, onde predominam fisionomias campestres, e outro do oeste paulista, onde predominam os cerradões (Durigan et al. 2003a; b). Existem áreas menores, floristicamente distintas, relacionadas a condições ambientais particulares, como o extremo nordeste de São Paulo, nos municípios de Rifaina e Pedregulho (Durigan et al. 2002; 2003b). Nesses municípios ocorre alta proporção de espécies raras ou com distribuição restrita (Durigan et al. 2003a). É uma região de prioridade máxima de conservação por suas características ecológicas, político-sociais, e pelo tamanho e localização dos fragmentos nativos (Brito 1997), além de ter sido uma das áreas mais intensamente devastadas nos últimos 30 anos (Kronka et al. 1998).

Sob este panorama, o presente estudo contribui para o conhecimento da flora do cerrado paulista, apresentando uma listagem florística de duas regiões

\footnotetext{
Parte da Dissertação de Mestrado da primeira Autora

2 Universidade de São Paulo, Instituto de Biociências, C. Postal 11461, 05422-970 São Paulo, SP, Brasil

3 Autor para correspondência: denisesasaki@hotmail.com
} 
no município de Pedregulho, o Parque Estadual das Furnas do Bom Jesus e o distrito de Estreito. Ainda, a fim de contribuir para a compreensão da dissimilaridade de sua flora em relação aos demais remanescentes de cerrado paulista, são investigados neste estudo padrões de distribuição geográfica das espécies cuja ocorrência no Estado de São Paulo é restrita à região de Pedregulho.

\section{Material e métodos}

O município de Pedregulho localiza-se no extremo nordeste do Estado de São Paulo, nas coordenadas aproximadas de $20^{\circ} 14^{\prime} 55^{\prime \prime}$ de latitude sul e $47^{\circ} 28^{\prime} 48^{\prime \prime}$ de longitude oeste (Fig. 1). O clima é do tipo Cwa de Köppen (1948), Temperado Moderado Chuvoso, com chuvas periódicas, inverno seco não rigoroso e temperatura mais quente superior a $22{ }^{\circ} \mathrm{C}$; ou do tipo Tropical Subquente Semi-Úmido, com período seco de 4 a 5 meses (Nimer 1989).

Pedregulho está situado em unidades geológicas distintas. A maior parte encontra-se sobre sedimentos paleozóicos e mesozóicos da Bacia Sedimentar do Paraná (Almeida 1964; Moreira \& Camelier 1977; Ross \& Moroz 1997), mais precisamente na porção norte da formação basáltico-arenítica mais ou menos contínua que separa a Depressão Periférica do Planalto Ocidental Paulista (Planaltos Cuestiformes ou Cuestas Basálticas) (Ross \& Moroz 1997). Entretanto, uma faixa estreita marginal ao rio Grande, que inclui o distrito de Estreito e o município de Rifaina, situa-se sobre rochas pré-cambrianas do proterozóico médio, pertencentes ao grupo Canastra (Almeida et al. 1981; Gatto et al. 1983) (Fig. 1). Essas rochas, expostas devido à remoção de litologias jurássico-cretáceas pelo entalhamento do rio, constituem-se de litologias ocorrentes na região dos Planaltos da Canastra, localizados em Minas Gerais, e que se estendem até as imediações do Complexo Hidrelétrico de Furnas em São Paulo (Gatto et al. 1983).

O Parque Estadual das Furnas do Bom Jesus, criado em 1989, é a única unidade de conservação do nordeste de São Paulo. Sua área é de 2.069,06 ha e engloba parte da bacia do córrego do Pedregulho (Branco et al. 1991; Barbosa \& Nunes 2001). A maior parte do Parque é coberta por capoeiras baixas e florestas estacionais semidecíduas primárias (Barbosa \& Nunes 2001), que ocupam as escarpas das furnas e o fundo do vale do córrego do Pedregulho. Nas regiões mais elevadas, existem áreas de cerrado com diferentes fisionomias: campo sujo, campo cerrado e cerrado sensu stricto.

$\mathrm{O}$ distrito de Estreito situa-se na margem sul do lago da usina hidrelétrica Luiz Carlos Barreto Carvalho, no rio Grande. Em suas margens existem áreas de vegetação bem preservadas, ocasionalmente atingidas por incêndios, que cobrem encostas pedregosas, de declividade suave a bastante acidentada. A vegetação apresenta um gradiente de fisionomias de campo sujo, campo cerrado, cerrado sensu stricto e floresta estacional semidecídua. Estreito está localizado sobre

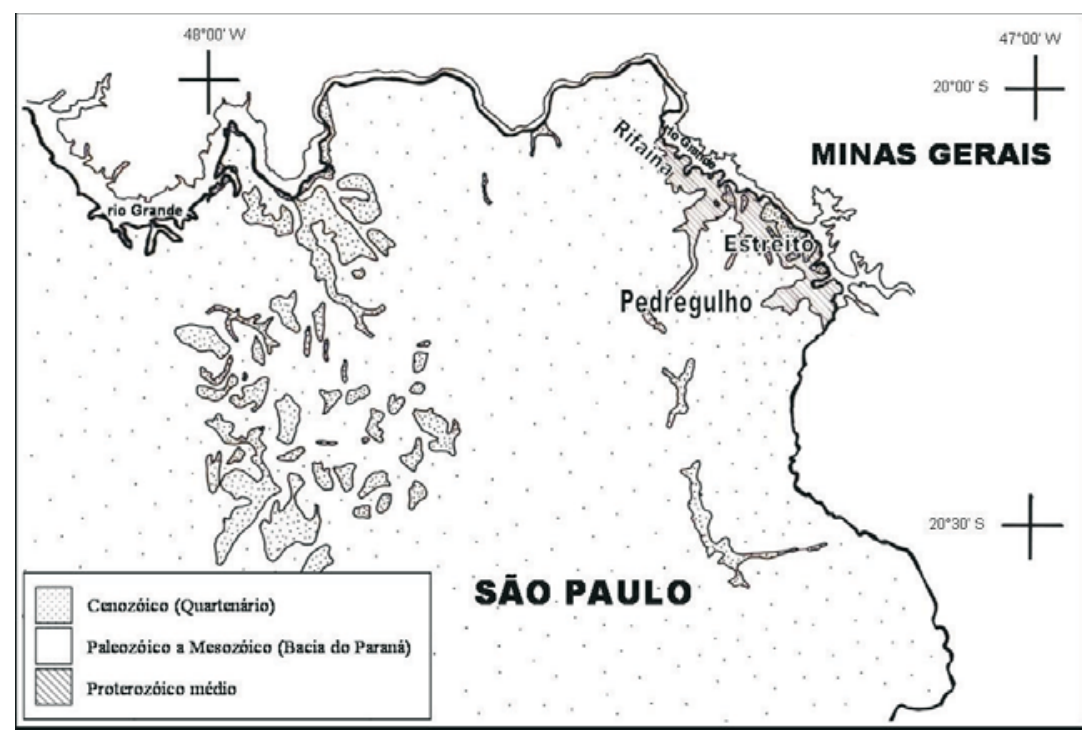

Figura 1. Mapa geológico do extremo nordeste do Estado de São Paulo, Brasil. Adaptado de Almeida et al. (1981). 
a faixa marginal ao rio Grande onde estão expostas litologias de origem pré-cambriana, pertencente ao grupo Canastra (Almeida et al. 1981).

Foram amostradas quatro parcelas de $50 \times 50 \mathrm{~m}$, totalizando um hectare, duas no Parque Estadual das Furnas do Bom Jesus (áreas 1 e 2) e duas no distrito de Estreito (áreas 3 e 4). Cada parcela apresenta características distintas (Tab. 1). Para uma amostragem mais completa, coletou-se também em suas adjacências.

Foram realizadas 14 expedições de coleta mensais de aproximadamente quatro dias cada, entre abril/2003 e abril/2004, e em outubro/2004. As parcelas foram percorridas de forma assistemática, e coletaram-se e herborizam-se as angiospermas férteis. O material botânico testemunho encontra-se depositado nos herbários citados na Tab. 2. A classificação taxonômica segue APG II (2003). Em Compositae, optou-se pela classificação sensu lato dos gêneros Vernonia Schreb. e Eupatorium L.

As composições florísticas de cada área foram comparadas entre si e realizou-se uma análise de agrupamento por UPGMA (Unweighted Pair Group Method with Arithmetic Average) entre as quatro áreas. Para isso, foram utilizados o coeficiente de Sorensen e o programa MVSP versão 3.1. (Kovach Computer Services). Para análise dos padrões fitogeográficos foram excluídas espécies de distribuição muito ampla (cosmopolita, tropical ou neotropical), exóticas, que fossem comuns às áreas de cerrado de todo Brasil, ou que se distribuíssem por outras localidades do Estado de São Paulo. Dessa forma, foram selecionadas espécies de distribuição mais restrita ou cuja ocorrência no Estado de São Paulo se restringe à região de Pedregulho. Dados de distribuição foram obtidos de trabalhos florísticos de regiões de São Paulo, Minas Gerais e Bahia, de revisões taxonômicas, de bancos informatizados dos herbários
F, IAC, MO, NY, S, U e US, e de coleções dos herbários SPF e UEC.

\section{Resultados}

Foram encontradas dentro das parcelas 65 famílias e 379 espécies e mais seis famílias e 64 espécies nas adjacências das parcelas, totalizando 71 famílias e 443 espécies (Tab. 2).

As famílias com maior número de espécies são Compositae (63 espécies), Leguminosae (49 spp.), Gramineae (38 spp.), Melastomataceae (22 spp.), Malpighiaceae (20 spp.), Cyperaceae (18 spp.) e Rubiaceae (16 spp.) (Fig. 2), representando 51\% do total coletado. Cinco famílias apresentaram três espécies, 11 famílias duas espécies e 28 famílias uma única espécie. Dessa forma, aproximadamente $62 \%$ das famílias (44) englobam somente $15 \%$ das espécies (65 spp.).

Das 443 espécies coletadas em Pedregulho, 289 são ervas ou subarbustos e 154 arbustos ou árvores. A proporção aproximada entre o componente herbáceosubarbustivo e o arbustivo-arbóreo é de 2:1 Apesar das diferentes fisionomias, nas áreas 1 e 4, 60\% das espécies são ervas ou subarbustos e $40 \%$ arbustos ou árvores, e nas áreas 2 e 3, são $63 \%$ e 37\%, respectivamente.

As parcelas nas quais registrou-se maior número de espécies são, em ordem decrescente, as da área 1 (198 espécies), área 2 (156 espécies), área 4 (116 espécies) e área 3 (98 espécies). Somando-se as espécies coletadas nas adjacências, totalizaram-se os seguintes números de espécies: 206 na área 1, 228 na área 2, 121 na área 3 e 127 na área 4.

As áreas 1 e 2, no P.E. das Furnas do Bom Jesus, totalizam 346 espécies e as áreas 3 e 4, em Estreito, 180 espécies. Foram registradas 86 espécies em comum

Tabela 1. Caracterização das quatro áreas anaisadas no município de Pedregulho, SP, Brasil.

\begin{tabular}{|c|c|c|c|c|}
\hline \multirow[b]{2}{*}{ Localidade } & Área 1 & Área 2 & Área 3 & Área 4 \\
\hline & $\begin{array}{c}\text { P.E. das Furnas do } \\
\text { Bom Jesus }\end{array}$ & $\begin{array}{l}\text { P.E. das Furnas do } \\
\text { Bom Jesus }\end{array}$ & Distrito de Estreito & Distrito de Estreito \\
\hline \multirow[t]{2}{*}{ Coordenadas } & $20^{\circ} 11 ' 50 ’ \mathrm{~S}$ & $20^{\circ} 14^{\prime} 52 ” \mathrm{~S}$ & $20^{\circ} 09^{\prime} 52 ’ \mathrm{~S}$ & $20^{\circ} 09^{\prime} 28^{\prime \prime} \mathrm{S}$ \\
\hline & $47^{\circ} 25^{\prime} 10^{\prime \prime} \mathrm{W}$ & $47^{\circ} 27^{\prime} 37^{\prime \prime} \mathrm{W}$ & $47^{\circ} 16^{\prime} 23^{\prime \prime} \mathrm{W}$ & $47^{\circ} 16^{\prime} 38^{\prime \prime} \mathrm{W}$ \\
\hline Altitude & $870-940 \mathrm{~m}$ & $977-1.000 \mathrm{~m}$ & $713-727 \mathrm{~m}$ & $711-715 \mathrm{~m}$ \\
\hline Declividade do terreno & $55^{\circ}$ & $5^{\circ}$ & $16^{\circ}$ & $12^{\circ}$ \\
\hline Fisionomia & campo cerrado a cerrado s.s. & campo sujo & campo sujo a campo cerrado & campo cerrado \\
\hline Solo & arenoso & areno-pedregoso & areno-pedregoso & areno-pedregoso \\
\hline Afloramentos rochosos & areníticos & ausentes & quartzíticos & quartzíticos \\
\hline Locais úmidos & ausentes & ausentes & brejo temporário & vala temporária \\
\hline
\end{tabular}


Tabela 2. Espécies encontradas nas áreas 1, 2, 3 e 4 em Pedregulho, SP, Brasil, e informações sobre o hábito (erv $=$ erva, sub $=$ subarbusto, $\mathrm{arb}=$ arbusto, árv $=$ árvore $)$ e material voucher $(\mathrm{DS}=$ Sasaki, $\mathrm{MF}=$ Farinaccio $) . \mathrm{x}=$ coletada dentro da parcela, $\mathrm{f}=$ coletada fora da parcela.

\begin{tabular}{|c|c|c|c|c|c|c|}
\hline Famílias/Espécies & Hábito & 1 & 2 & 3 & 4 & Voucher \\
\hline \multicolumn{7}{|l|}{ ACANTHACEAE } \\
\hline Ruellia geminiflora Kunth. & sub & & & $\mathrm{f}$ & & DS 824 \\
\hline Ruellia sp. & arb & $\mathrm{x}$ & & & & DS 1056 \\
\hline \multicolumn{7}{|l|}{ AMARANTHACEAE } \\
\hline Gomphrena prostrata Mart. & sub & $\mathrm{x}$ & $\mathrm{x}$ & $\mathrm{x}$ & $\mathrm{x}$ & DS 410 \\
\hline Gomphrena virgata Mart. & sub & & $\mathrm{x}$ & & & DS 643 \\
\hline AMARANTHACEAE sp. & sub & $\mathrm{x}$ & & & & DS 402 \\
\hline \multicolumn{7}{|l|}{ Anacardiaceae } \\
\hline Anacardium humile A. St.-Hil. & arb & & $\mathrm{f}$ & & & DS 776 \\
\hline Lithraea molleoides (Vell.) Engl. & árv & $\mathrm{x}$ & $\mathrm{f}$ & & & DS 680 \\
\hline \multicolumn{7}{|l|}{ ANNONACEAE } \\
\hline Annona coriacea Mart. & arb & & $\mathrm{f}$ & & & MF 549 \\
\hline A. cornifolia A. St.-Hil. & arb & $\mathrm{x}$ & & & & DS 866 \\
\hline Duguetia furfuracea (A. St.-Hil.) Saff. & arb & & $\mathrm{f}$ & & & DS 90 \\
\hline \multicolumn{7}{|l|}{ APOCYNACEAE } \\
\hline Aspidosperma macrocarpon Mart. & árv & & & $\mathrm{x}$ & $\mathrm{x}$ & DS 648 \\
\hline A. tomentosum Mart. & árv & $\mathrm{x}$ & & & $\mathrm{x}$ & DS 1065 \\
\hline Barjonia erecta (Vell.) K. Schum. & sub & $\mathrm{x}$ & & $\mathrm{x}$ & & DS 1008 \\
\hline B. laxa Malme & erv & & $\mathrm{x}$ & & & DS 845 \\
\hline Blepharodon nitidum (Vell.) J.F. Macbr. & erv & & $\mathrm{x}$ & & & DS 956 \\
\hline Hancornia speciosa Gomes & árv & & & $\mathrm{x}$ & $\mathrm{x}$ & DS 735 \\
\hline Himatanthus obovatus (Müll. Arg.) Woodson & árv & & & $\mathrm{x}$ & $\mathrm{x}$ & DS 375 \\
\hline Macrosiphonia longiflora (Desf.) Müll. Arg. & sub & & $\mathrm{x}$ & & & DS 769 \\
\hline M. velame (A. St.-Hil.) Müll. Arg. & sub & $\mathrm{x}$ & & & & DS 927 \\
\hline Mandevilla illustris (Vell.) Woodson & erv & & $\mathrm{f}$ & & & DS 771 \\
\hline M. pohliana (Stadelm.) A.H. Gentry & sub & $\mathrm{f}$ & $\mathrm{x}$ & & & DS 837 \\
\hline M. tenuifolia (J.C. Mikan) Woodson & erv & & & $\mathrm{x}$ & $\mathrm{x}$ & DS 852 \\
\hline Odontadenia lutea (Vell.) Markgr. & arb & & $\mathrm{x}$ & & $\mathrm{x}$ & DS 566 \\
\hline Oxypetalum capitatum Mart. & sub & $\mathrm{x}$ & $\mathrm{f}$ & & & MF 538 \\
\hline Rhodocalyx rotundifolius Müll. Arg. & sub & & $\mathrm{x}$ & & & DS 767 \\
\hline \multicolumn{7}{|l|}{ ARALIACEAE } \\
\hline Schefflera macrocarpa (Cham. \& Schltdl.) Frodin & árv & $\mathrm{x}$ & $\mathrm{x}$ & & & DS 89 \\
\hline \multicolumn{7}{|l|}{ ARISTOLOCHIACEAE } \\
\hline Aristolochia arcuata Mast. & erv & & & & $\mathrm{x}$ & DS 340 \\
\hline \multicolumn{7}{|l|}{ BIGNONIACEAE } \\
\hline Arrabidaea brachypoda (DC.) Bureau & arb & & $\mathrm{x}$ & & & DS 1021 \\
\hline Jacaranda rufa Silva Manso & arb & & & & $\mathrm{x}$ & DS 657 \\
\hline Tabebuia aurea (Silva Manso) Benth. \& Hook.f. ex S. Moore & árv & $\mathrm{x}$ & & & & DS 670 \\
\hline T. ochracea (Cham.) Standley & árv & $\mathrm{x}$ & & & $\mathrm{x}$ & DS 649 \\
\hline Zeyheria montana Mart. & arb & & $\mathrm{x}$ & & & DS 162 \\
\hline \multicolumn{7}{|l|}{ BIXACEAE } \\
\hline Cochlospermum regium (Schrank) Pilg. & arb & $\mathrm{f}$ & & & & DS 679 \\
\hline \multicolumn{7}{|l|}{ BORAGINACEAE } \\
\hline Cordia corymbosa Willd. ex Roem. \& Schult. & arb & & $\mathrm{f}$ & & & DS 148 \\
\hline \multicolumn{7}{|l|}{ BROMELIACEAE } \\
\hline Dyckia aff. linearifolia Baker & erv & $\mathrm{x}$ & $\mathrm{x}$ & $\mathrm{x}$ & $\mathrm{x}$ & DS 520 \\
\hline \multicolumn{7}{|l|}{ BURMANNIACEAE } \\
\hline Burmannia capitata (Walter ex J.F. Gmel.) Mart. & erv & & & $\mathrm{x}$ & & DS 482 \\
\hline B. flava Mart. & erv & & & $\mathrm{x}$ & & DS 470 \\
\hline \multicolumn{7}{|l|}{ CAMPANULACEAE } \\
\hline Lobelia camporum Pohl & erv & $\mathrm{f}$ & & & & DS 108 \\
\hline \multicolumn{7}{|l|}{ CARYOCARACEAE } \\
\hline Caryocar brasiliense Cambess. & árv & $\mathrm{x}$ & $\mathrm{x}$ & $\mathrm{x}$ & $\mathrm{x}$ & DS 693 \\
\hline
\end{tabular}


Tabela 2 (continuação)

\begin{tabular}{|c|c|c|c|c|c|c|}
\hline Famílias/Espécies & Hábito & 1 & 2 & 3 & 4 & Voucher \\
\hline \multicolumn{7}{|l|}{ CELASTRACEAE } \\
\hline Peritassa campestris (Cambess.) A.C. Sm. & arb & & $\mathrm{x}$ & & & DS 905 \\
\hline \multicolumn{7}{|l|}{ CHRYSOBALANACEAE } \\
\hline Hirtella gracilipes (Hook.f.) Prance & árv & $\mathrm{f}$ & & & & DS 1063 \\
\hline \multicolumn{7}{|l|}{ COMBRETACEAE } \\
\hline Terminalia argentea (Cambess.) Mart. & árv & $\mathrm{x}$ & $\mathrm{f}$ & & & DS 496 \\
\hline \multicolumn{7}{|l|}{ COMPOSITAE } \\
\hline Achyrocline satureioides (Lam.) DC. & arb & $\mathrm{x}$ & $\mathrm{X}$ & & & DS 613 \\
\hline Acmella bellidioides (Smith) L.K. Jansen & sub & $\mathrm{x}$ & & & & DS 810 \\
\hline Angelphytum arnottii (Baker) H. Rob. & erv & & $\mathrm{x}$ & & & DS 462 \\
\hline Aspilia foliacea Baker & erv & & $\mathrm{x}$ & & & DS 756 \\
\hline Baccharis camporum DC. & sub & & $\mathrm{f}$ & & & DS 124 \\
\hline B. dracunculifolia DC. & arb & $\mathrm{x}$ & $\mathrm{X}$ & & & DS 960 \\
\hline B. polyphylla Baker & arb & & $\mathrm{x}$ & & & DS 127 \\
\hline Bidens gardneri Baker & erv & $\mathrm{x}$ & & & & DS 508 \\
\hline B. graveolens Gardner & arb & $\mathrm{x}$ & & & & DS 975 \\
\hline Calea graminifolia Sch. Bip. ex Krasch. & erv & & & $\mathrm{x}$ & & DS 348 \\
\hline C. lantanoides Gardner & sub & $\mathrm{x}$ & & & & DS 967 \\
\hline C. serrata Less. & arb & & & & $\mathrm{x}$ & DS 345 \\
\hline Chaptalia integerrima (Vell.) Burkart & erv & $\mathrm{x}$ & & & & DS 808 \\
\hline Chresta scapigera (Less.) Gardner & sub & $\mathrm{x}$ & $\mathrm{x}$ & & $\mathrm{x}$ & DS 525 \\
\hline C. sphaerocephala DC. & sub & $\mathrm{X}$ & & & $\mathrm{x}$ & DS 565 \\
\hline Chrysanthellum tuberculatum (Hook. \& Arn.) Cabrera & erv & $\mathrm{x}$ & & & & DS 973 \\
\hline Dasyphyllum sprengelianum (Gardner) Cabrera & arb & $\mathrm{x}$ & $\mathrm{x}$ & & & DS 467 \\
\hline Elephantopus micropappus Less. & erv & & $\mathrm{X}$ & & & DS 954 \\
\hline Eremanthus matogrossensis Kunth & árv & & $\mathrm{x}$ & $\mathrm{f}$ & $\mathrm{x}$ & DS 527 \\
\hline E. seidelii MacLeish\& H. Schumach. & árv & & & $\mathrm{X}$ & $\mathrm{x}$ & DS 529 \\
\hline Eupatorium amygdalinum Lam. & sub & & $\mathrm{x}$ & & & DS 642 \\
\hline E. calamocephalum (Baker) Hieron. & sub & & $\mathrm{X}$ & & & DS 305 \\
\hline E. dentatum Gardner & sub & $\mathrm{x}$ & & & & DS 676 \\
\hline E. dimorpholepis Baker & sub & $\mathrm{x}$ & $\mathrm{X}$ & & $\mathrm{x}$ & DS 900 \\
\hline E. ferrugineum Gardner & sub & & & & $\mathrm{x}$ & DS 324 \\
\hline E. kleinioides Kunth & erv & $\mathrm{x}$ & $\mathrm{x}$ & & & DS 302 \\
\hline E. leptophlebium (B.L. Rob.) Steyerm. & sub & & $\mathrm{f}$ & & & DS 155 \\
\hline E. cf. oxylepis DC. & arb & $\mathrm{x}$ & $\mathrm{f}$ & $\mathrm{X}$ & $\mathrm{x}$ & DS 317 \\
\hline E. squalidum DC. & sub & & $\mathrm{x}$ & & & DS 310 \\
\hline Eupatorium sp. & erv & & & $\mathrm{x}$ & $\mathrm{x}$ & DS 483 \\
\hline Gochnatia barrosii Cabrera & arb & & $\mathrm{X}$ & & & DS $595 b$ \\
\hline Lychnophora ericoides Mart. & arb & & & $\mathrm{x}$ & & DS 379 \\
\hline Mikania triphylla Spreng. ex Baker & sub & & & & $\mathrm{x}$ & DS 795 \\
\hline Orthopappus angustifolius (Sw.) Gleason & erv & $\mathrm{x}$ & & & & DS 913 \\
\hline Piptocarpha rotundifolia (Less.) Baker & arb & & $\mathrm{f}$ & & & DS 125 \\
\hline Pterocaulon balansae Chodat & sub & & $\mathrm{x}$ & & & DS 300 \\
\hline Riencourtia oblongifolia Gardner & arb & & $\mathrm{x}$ & & & DS 877 \\
\hline Senecio grisebachii Baker & sub & & $\mathrm{f}$ & & & DS 121 \\
\hline Stevia hilarii B.L. Rob. & erv & $\mathrm{x}$ & & & & DS 383 \\
\hline Strophopappus speciosus (Less.) R. Esteves & arb & $\mathrm{x}$ & $\mathrm{x}$ & & & DS 610 \\
\hline Trixis glutinosa Don & erv & $\mathrm{x}$ & $\mathrm{x}$ & & & DS 574 \\
\hline Vernonia ammophila Gardner & sub & $\mathrm{x}$ & & & & DS 510 \\
\hline V. apiculata Mart. ex DC. & erv & & $\mathrm{f}$ & & & DS 156 \\
\hline V. bardanoides Less. & arb & & $\mathrm{x}$ & & & DS 299 \\
\hline V. buddleiifolia Mart. ex DC. & sub & & & & $\mathrm{x}$ & DS 986 \\
\hline V. coriacea Less. & arb & & $\mathrm{x}$ & & & DS 297 \\
\hline V. dura Mart. ex DC. & sub & $\mathrm{x}$ & $\mathrm{x}$ & & & DS 491 \\
\hline V. elegans Gardner & sub & $\mathrm{x}$ & & & & DS 399 \\
\hline V. ferruginea Less. & arb & & $\mathrm{x}$ & & & DS 605 \\
\hline V. geminata Kunth. & arb & $\mathrm{x}$ & & & & DS 417 \\
\hline
\end{tabular}


Tabela 2 (continuação)

\begin{tabular}{|c|c|c|c|c|c|c|}
\hline Famílias/Espécies & Hábito & 1 & 2 & 3 & 4 & Voucher \\
\hline Vernonia herbacea (Vell.) Rusby & erv & $\mathrm{x}$ & & & & DS 820 \\
\hline V. obtusata Less. & arb & & $\mathrm{x}$ & $\mathrm{x}$ & $\mathrm{x}$ & DS 988 \\
\hline V. rosea Mart. ex DC. & sub & & $\mathrm{x}$ & & & DS 906 \\
\hline V. rufogrisea A. St.-Hil. & arb & & & $\mathrm{x}$ & $\mathrm{x}$ & DS 367 \\
\hline V. schwenkiifolia Mart. & sub & & $\mathrm{x}$ & $\mathrm{x}$ & & DS 480 \\
\hline V. simplex Less. & erv & $\mathrm{x}$ & & $\mathrm{x}$ & $\mathrm{x}$ & DS 784 \\
\hline V. tragiifolia DC. & erv & $\mathrm{x}$ & $\mathrm{x}$ & & & DS 839 \\
\hline Vernonia sp. nov. & sub & & & $\mathrm{x}$ & & DS 558 \\
\hline Viguiera bracteata Gardner & sub & & & & $\mathrm{x}$ & DS 937 \\
\hline V. discolor Baker & sub & $\mathrm{x}$ & $\mathrm{f}$ & & & DS 823 \\
\hline Wedelia puberula DC. & sub & & $\mathrm{x}$ & & & DS 843 \\
\hline W. subvelutina $\mathrm{DC}$. & sub & $\mathrm{x}$ & & & & DS 911 \\
\hline Wunderlichia mirabilis Riedel ex Baker & árv & & & & $\mathrm{f}$ & DS 651 \\
\hline \multicolumn{7}{|l|}{ CONNARACEAE } \\
\hline Rourea induta Planch. & árv & $\mathrm{x}$ & & $\mathrm{x}$ & $\mathrm{x}$ & DS 799 \\
\hline Connaraceae sp. & árv & & & & $\mathrm{x}$ & DS 570 \\
\hline \multicolumn{7}{|l|}{ CONVOLVULACEAE } \\
\hline Evolvulus sericeus $\mathrm{Sw}$. & erv & $\mathrm{x}$ & & & & DS 807 \\
\hline Ipomoea procumbens Mart. ex Choisy & erv & & $\mathrm{x}$ & & & DS 949 \\
\hline I. procurrens Meisn. & sub & & $\mathrm{x}$ & & & DS 311 \\
\hline I. virgata Meisn. & arb & & $\mathrm{f}$ & & & DS 87 \\
\hline Jacquemontia selloi (Meisn.) Hallier f. & erv & $\mathrm{x}$ & & & & DS 817 \\
\hline J. sphaerostigma (Cav.) Rusby & erv & & & & $\mathrm{x}$ & DS 319 \\
\hline J. spiciflora (Choisy) Hallier f. & arb & & $\mathrm{f}$ & & & DS 112 \\
\hline Merremia tomentosa (Choisy) Hallier f. & arb & & $\mathrm{x}$ & & & DS 313 \\
\hline \multicolumn{7}{|l|}{ CUCURBITACEAE } \\
\hline Cayaponia espelina (Silva Manso) Cogn. & sub & & $\mathrm{f}$ & & & MF 548 \\
\hline \multicolumn{7}{|l|}{ CYPERACEAE } \\
\hline Bulbostylis capillaris (L.) C.B. Clarke & erv & $\mathrm{x}$ & & & & DS 805 \\
\hline B. fendleri C.B. Clarke & erv & $\mathrm{x}$ & & & & DS 422 \\
\hline B. hirtella (Schrad. ex Schult.) Nees ex Urb. & erv & & & & $\mathrm{f}$ & DS 191 \\
\hline B. jacobinae (Steud.) Lindm. & erv & $\mathrm{x}$ & & & & DS 984 \\
\hline B. junciformis (Kunth) C.B. Clarke & erv & $\mathrm{x}$ & $\mathrm{x}$ & $\mathrm{x}$ & $\mathrm{x}$ & DS 309 \\
\hline B. paradoxa (Spreng.) Lindm. & erv & & & $\mathrm{x}$ & & DS 783 \\
\hline B. sphaerocephala (Boeckeler) C.B. Clarke & erv & & $\mathrm{f}$ & & $\mathrm{x}$ & DS 796 \\
\hline B. vestita (Kunth) C.B. Clarke & erv & & $\mathrm{x}$ & & & DS 881 \\
\hline Bulbostylis sp. 1 & erv & & & $\mathrm{f}$ & $\mathrm{f}$ & DS 188 \\
\hline Bulbostylis sp. 2 & erv & & & & $\mathrm{f}$ & DS 146 \\
\hline Cyperus niger Ruiz \& Pav. & erv & & $\mathrm{f}$ & $\mathrm{f}$ & & DS 207 \\
\hline C. surinamensis Rottb. & erv & & & & $\mathrm{x}$ & DS 938 \\
\hline Rhynchospora armerioides J. Presl \& C. Presl & erv & & & & $\mathrm{f}$ & DS 196 \\
\hline R. consanguinea (Kunth) Boeckeler. & erv & & & $\mathrm{x}$ & & DS 355 \\
\hline R. globosa (Kunth) Roem. \& Schult. & erv & & & $\mathrm{x}$ & & DS 615 \\
\hline R. pallida (Nees) Steud. & erv & & $\mathrm{x}$ & & & DS 880 \\
\hline R. terminalis (Nees) Steud. & erv & & & $\mathrm{f}$ & $\mathrm{x}$ & DS 332 \\
\hline Scleria sp. & erv & $\mathrm{x}$ & & & $\mathrm{x}$ & DS 991 \\
\hline \multicolumn{7}{|l|}{ DILLENIACEAE } \\
\hline Curatella americana $\mathrm{L}$. & árv & $\mathrm{x}$ & $\mathrm{x}$ & & & DS 701 \\
\hline Davilla elliptica A. St.-Hil. & arb & $\mathrm{x}$ & $\mathrm{x}$ & & & DS 255 \\
\hline \multicolumn{7}{|l|}{ DROSERACEAE } \\
\hline Drosera hirtella A. St.- & erv & & $\mathrm{f}$ & & & DS 167 \\
\hline D. montana A. St.-Hil. & erv & $\mathrm{x}$ & $\mathrm{f}$ & & & DS 433 \\
\hline \multicolumn{7}{|l|}{ ERIOCAULACEAE } \\
\hline Actinocephalus polyanthus (Bong.) Sano & erv & & & $\mathrm{f}$ & & DS 210 \\
\hline Paepalanthus elongatus Körn. & erv & & & $\mathrm{f}$ & & DS 540 \\
\hline Syngonathus gracilis (Bong.) Ruhland & erv & & & $\mathrm{f}$ & & DS s.n. \\
\hline
\end{tabular}


Tabela 2 (continuação)

\begin{tabular}{|c|c|c|c|c|c|c|}
\hline Famílias/Espécies & Hábito & 1 & 2 & 3 & 4 & Voucher \\
\hline Syngonathus nitens (Bong.) Ruhland & erv & & $\mathrm{f}$ & $\mathrm{x}$ & & DS 236 \\
\hline \multicolumn{7}{|l|}{ ERYTHROXYLACEAE } \\
\hline Erythroxylum campestre A. St.-Hil. & arb & $\mathrm{x}$ & $\mathrm{x}$ & & & DS 678 \\
\hline E. suberosum A. St.-Hil. & arb & $\mathrm{x}$ & $\mathrm{x}$ & & $\mathrm{x}$ & DS 759 \\
\hline E. tortuosum Mart. & árv & & $\mathrm{x}$ & $\mathrm{x}$ & & DS 736 \\
\hline \multicolumn{7}{|l|}{ EUPHORBIACEAE } \\
\hline Bernardia hirsutissima (Baill.) Müll. Arg. & sub & $\mathrm{x}$ & & $\mathrm{x}$ & $\mathrm{x}$ & DS 562 \\
\hline Chamaesyce hyssopifolia (L.) Small & erv & $\mathrm{f}$ & & & & DS 982 \\
\hline C. potentilloides (Boiss.) Croizat & sub & $\mathrm{x}$ & & $\mathrm{x}$ & $\mathrm{x}$ & DS 338 \\
\hline C. selloi (Boiss.) Croizat & erv & $\mathrm{x}$ & $\mathrm{x}$ & & & DS 671 \\
\hline Croton antisyphiliticus Mart. & sub & & $\mathrm{x}$ & $\mathrm{x}$ & & DS 737 \\
\hline C. glandulosus $\mathrm{L}$. & sub & & & & $\mathrm{x}$ & DS 1032 \\
\hline C. grandivelum Baill. & sub & $\mathrm{x}$ & $\mathrm{f}$ & & & DS 928 \\
\hline C. sclerocalyx Müll. Arg. & erv & & $\mathrm{x}$ & & & DS 1051 \\
\hline Manihot gracilis Pohl & sub & & & $\mathrm{x}$ & & DS 733 \\
\hline M. triphylla Pohl & sub & & $\mathrm{x}$ & & & DS 754 \\
\hline Maprounea brasiliensis A. St.-Hil. & arb & & & & $\mathrm{x}$ & DS 798 \\
\hline Sebastiania bidentata (Mart.) Pax & sub & & $\mathrm{x}$ & & & DS 904 \\
\hline S. glandulosa (Mart.) Pax & sub & & & $\mathrm{x}$ & $\mathrm{x}$ & DS 888 \\
\hline \multicolumn{7}{|l|}{ GENTIANACEAE } \\
\hline Curtia tenella (Mart.) Cham. & erv & $\mathrm{x}$ & & $\mathrm{x}$ & $\mathrm{x}$ & DS 380 \\
\hline Deianira chiquitana Herzog & sub & & $\mathrm{x}$ & $\alpha$ & $\mathrm{x}$ & DS 308 \\
\hline D. pallescens Cham. \& Schltdl. & erv & & $\mathrm{x}$ & & & DS 460 \\
\hline Irlbachia speciosa (Cham. \& Schltdl.) Maas & sub & $\mathrm{x}$ & $\mathrm{x}$ & $\mathrm{x}$ & $\mathrm{x}$ & DS 347 \\
\hline \multicolumn{7}{|l|}{ GESNERIACEAE } \\
\hline Sinningia elatior (Kunth) Chautems & sub & $\mathrm{x}$ & & & & DS 979 \\
\hline \multicolumn{7}{|l|}{ GRAMINEAE } \\
\hline Andropogon carinatus Nees & erv & & $\mathrm{x}$ & & & DS 598 \\
\hline A. leucostachyus Kunth & erv & & $\mathrm{f}$ & $\mathrm{f}$ & & DS 246 \\
\hline Aristida riparia Trin. & erv & $\mathrm{x}$ & $\mathrm{x}$ & & & DS 315 \\
\hline Arthropogon villosus Nees & erv & & $\mathrm{x}$ & & & DS 1054 \\
\hline Axonopus aureus P. Beauv. & erv & & $\mathrm{x}$ & $\mathrm{x}$ & $\mathrm{x}$ & DS 307 \\
\hline A. brasiliensis (Spreng.) Kuhlm. & erv & & $\mathrm{x}$ & & & DS 755 \\
\hline A. suffultus (J.C. Mikan ex Trin.) Parodi & erv & & & $\mathrm{x}$ & $\mathrm{x}$ & DS 935 \\
\hline Ctenium cirrhosum (Nees) Kunth & erv & $\mathrm{x}$ & $\mathrm{x}$ & & & DS 298 \\
\hline Echinolaena inflexa (Poir.) Chase & erv & & $\mathrm{x}$ & $\mathrm{x}$ & $\mathrm{x}$ & DS 314 \\
\hline Eragrostis polytricha Nees & erv & & $\mathrm{x}$ & & & DS 450 \\
\hline Eragrostis solida Nees & erv & & $\mathrm{x}$ & & & DS 293 \\
\hline Hyparrhenia bracteata (Willd.) Stapf. & erv & $\mathrm{x}$ & & & & DS 499 \\
\hline H. rufa (Nees) Stapf. & erv & $\mathrm{x}$ & & & & DS 588 \\
\hline Hypogynium virgatum (Desv.) Dandy & erv & & $\mathrm{f}$ & & & DS 265 \\
\hline Ichnanthus inconstans (Trin. ex Nees) Döll. & erv & $\mathrm{x}$ & & & & DS 440 \\
\hline I. procurrens (Nees ex Trin.) Swallen & erv & & $\mathrm{x}$ & & & DS 844 \\
\hline Leptocoryphium lanatum (Kunth) Nees & erv & & $\mathrm{x}$ & & & DS 757 \\
\hline Loudetiopsis chrysothrix (Nees) Conert & erv & $\mathrm{x}$ & $\mathrm{x}$ & $\mathrm{x}$ & $\mathrm{x}$ & DS 333 \\
\hline Melinis minutiflora $\mathrm{P}$. Beauv. & erv & $\mathrm{x}$ & & & & DS 504 \\
\hline Mesosetum loliiforme (Hochst. ex Steud.) Chase & erv & & & & $\mathrm{x}$ & DS 922 \\
\hline Panicum cyanescens Nees ex Trin. & erv & & & $\mathrm{x}$ & & DS 485 \\
\hline P. olyroides Kunth & erv & $\mathrm{x}$ & $\mathrm{f}$ & & & DS 398 \\
\hline P. quadriglume (Döll) Hitchc. & erv & $\mathrm{x}$ & & & & DS 501 \\
\hline Paspalum erianthum Nees ex Trin. & erv & & $\mathrm{x}$ & & & DS 835 \\
\hline P. еисотит Nees ex Trin. & erv & & $\mathrm{x}$ & $\mathrm{x}$ & $\mathrm{x}$ & DS 296 \\
\hline P. gardnerianum Nees & erv & & & $\mathrm{x}$ & $\mathrm{x}$ & DS 337 \\
\hline$P$. aff. gardnerianum Nees & erv & $\mathrm{x}$ & $\mathrm{x}$ & & & DS 284 \\
\hline P. pilosum Lam. & erv & $\mathrm{x}$ & & & & DS s.n. \\
\hline P. polyphyllum Nees ex Trin. & erv & $\mathrm{x}$ & & $\mathrm{x}$ & & DS 413 \\
\hline P. stellatum Humb. \& Bonpl. ex Flüggé & erv & & $\mathrm{x}$ & & & DS 459 \\
\hline
\end{tabular}


Tabela 2 (continuação)

\begin{tabular}{|c|c|c|c|c|c|c|}
\hline Famílias/Espécies & Hábito & 1 & 2 & 3 & 4 & Voucher \\
\hline Rhynchelytrum repens (Willd.) C.E. Hubb. & erv & & $\mathrm{f}$ & & $\mathrm{x}$ & DS 528 \\
\hline Schizachyrium condensatum (Kunth) Nees & erv & & $\mathrm{x}$ & & & DS 456 \\
\hline S. sanguineum (Retz.) Alston & erv & & $\mathrm{x}$ & & $\mathrm{x}$ & DS 343 \\
\hline Sorghastrum sp. & erv & $\mathrm{x}$ & $\mathrm{x}$ & & & DS 952 \\
\hline Sporobolus aeneus (Trin.) Kunth & erv & & & & $\mathrm{x}$ & DS s.n. \\
\hline Trachypogon plumosus (Humb. \& Bonpl. ex Willd.) Nees & erv & & & & $\mathrm{x}$ & DS 339 \\
\hline T. spicatus (L.f.) Kuntze & erv & & & & $\mathrm{x}$ & DS 923 \\
\hline Urochloa brizantha (Hochst. ex A. Rich.) Webster & erv & & & & $\mathrm{x}$ & DS 335 \\
\hline \multicolumn{7}{|l|}{ GUTTIFERAE } \\
\hline Kielmeyera coriacea Mart. \& Zucc. & árv & $\mathrm{x}$ & $\mathrm{x}$ & & $\mathrm{x}$ & DS 794 \\
\hline K. corymbosa Mart. & arb & $\mathrm{x}$ & & & & DS 708 \\
\hline K. pumila Pohl & arb & & & $\mathrm{f}$ & & DS 223 \\
\hline K. rubriflora Cambess. & arb-árv & $\mathrm{x}$ & & $\mathrm{x}$ & $\mathrm{x}$ & DS 322 \\
\hline \multicolumn{7}{|l|}{ IRIDACEAE } \\
\hline Cipura paludosa Aubl. & erv & & $\mathrm{f}$ & & & DS 82 \\
\hline Trimezia juncifolia (Klatt) Benth. \& Hook.f. & erv & $\mathrm{x}$ & $\mathrm{x}$ & $\mathrm{x}$ & & DS 472 \\
\hline T. lutea (Klatt) R.C. Foster & erv & & $\mathrm{f}$ & & & DS 83 \\
\hline Iridaceae sp. 1 & erv & & $\mathrm{x}$ & & & DS 389 \\
\hline Iridaceae sp. 2 & erv & $\mathrm{x}$ & & & & DS 914 \\
\hline \multicolumn{7}{|l|}{ LABIATAE } \\
\hline Eriope crassipes Benth. & erv & $\mathrm{x}$ & & & & DS 814 \\
\hline Hypenia sp. & sub & $\mathrm{x}$ & & $\mathrm{x}$ & $\mathrm{x}$ & DS 385 \\
\hline Hyptidendron canum (Pohl ex Benth.) Harley & árv & $\mathrm{x}$ & & & & DS 628 \\
\hline Hyptis carpinifolia Benth. & arb & $\mathrm{x}$ & & & & DS 640 \\
\hline H. lantanifolia Poit. & erv & & $\mathrm{f}$ & & & DS 117 \\
\hline H. lutescens Pohl ex Benth. & arb & $\mathrm{x}$ & & & & DS 977 \\
\hline H. rugosa Benth. & arb & $\mathrm{x}$ & $\mathrm{x}$ & & & DS 575 \\
\hline H. sericea Benth. & sub & $\mathrm{x}$ & $\mathrm{x}$ & & & DS 407 \\
\hline H. virgata Benth. & erv & & $\mathrm{x}$ & & & DS 770 \\
\hline Rhabdocaulon denudatum (Benth.) Epling & erv & $\mathrm{x}$ & $\mathrm{f}$ & & & DS 394 \\
\hline Salvia minarum Briq. & sub & $\mathrm{x}$ & & & & DS 404 \\
\hline S. tomentella Pohl & sub & $\mathrm{x}$ & $\mathrm{f}$ & & & DS 815 \\
\hline \multicolumn{7}{|l|}{ LAURACEAE } \\
\hline Cassytha filiformes $\mathrm{L}$. & erv & $\mathrm{x}$ & $\mathrm{x}$ & & & DS 436 \\
\hline \multicolumn{7}{|l|}{ LEGUMINOSAE } \\
\hline Acosmium dasycarpum (Vogel) Yak. & árv & $\mathrm{x}$ & $\mathrm{f}$ & & & DS 856 \\
\hline Aeschynomene paniculata Willd. & arb & $\mathrm{x}$ & $\mathrm{x}$ & & $\mathrm{x}$ & DS 326 \\
\hline Andira humilis Mart. ex Benth. & árv & & & & $\mathrm{x}$ & DS 1058 \\
\hline Bauhinia rufa Steud. & arb & & $\mathrm{x}$ & & & DS 840 \\
\hline Calliandra dysantha Benth. & sub & & & $\mathrm{x}$ & $\mathrm{x}$ & DS 778 \\
\hline Camptosema coriaceum (Nees \& C. Mart.) Benth. & arb & $\mathrm{x}$ & & & & DS 589 \\
\hline C. scarlatinum (Mart. ex Benth.) Burk. & arb & $\mathrm{x}$ & & & & DS 818 \\
\hline Centrosema angustifolium (Kunth) Benth. & erv & & $\mathrm{f}$ & & & DS 141 \\
\hline Chamaecrista basifolia (Vogel) H.S. Irwin \& Barneby & erv & $\mathrm{x}$ & & & & DS 493 \\
\hline C. cathartica (Mart.) H.S. Irwin \& Barneby var. cathartica & arb & $\mathrm{x}$ & $\mathrm{x}$ & $\mathrm{x}$ & $\mathrm{x}$ & DS 514 \\
\hline C. desvauxii (Collad.) Killip & sub & & $\mathrm{x}$ & $\mathrm{f}$ & & DS 290 \\
\hline C. kunthiana (Schltdl. \& Cham.) H.S. Irwin \& Barneby & erv & & & $\mathrm{f}$ & & DS 208 \\
\hline $\begin{array}{l}\text { C. ochnacea (Vogel) H.S. Irwin \& Barneby var. speluncae } \\
\text { (H.S. Irwin \& Barneby) H.S. Irwin \& Barneby }\end{array}$ & sub & & & $\mathrm{x}$ & $\mathrm{x}$ & DS 346 \\
\hline C. rotundifolia (Pers.) Greene & sub & & $\mathrm{x}$ & & & DS 1040 \\
\hline C. setosa (Vogel) H.S. Irwin \& Barneby var. setosa & arb & $\mathrm{x}$ & & & & DS 498 \\
\hline Clitoria guianensis (Aubl.) Benth. & sub & $\mathrm{x}$ & $\mathrm{x}$ & & & DS 822 \\
\hline Crotalaria nitens Kunth & sub & $\mathrm{x}$ & $\mathrm{x}$ & & & DS 388 \\
\hline C. pallida Aiton & sub & & $\mathrm{f}$ & & & DS 142 \\
\hline Desmodium adscendes (Sw.) DC. & sub & $\mathrm{x}$ & & & & DS 802 \\
\hline D. barbatum (L.) Benth. & sub & & $\mathrm{f}$ & & & DS 135 \\
\hline
\end{tabular}


Tabela 2 (continuação)

\begin{tabular}{|c|c|c|c|c|c|c|}
\hline Famílias/Espécies & Hábito & 1 & 2 & 3 & 4 & Voucher \\
\hline Desmodium incanum (Sw.) DC. & sub & $\mathrm{x}$ & & & & DS 806 \\
\hline D. pachyrhizum Vogel & erv & $\mathrm{x}$ & & & & DS 972 \\
\hline Dimorphandra mollis Benth. & árv & $\mathrm{x}$ & $\mathrm{x}$ & & & DS 854 \\
\hline Eriosema crinitum (Kunth) G. Don & sub & & $\mathrm{f}$ & & & DS 773 \\
\hline Galactia decumbens (Benth.) Chodat \& Hassl. & erv & & $\mathrm{x}$ & & & DS 902 \\
\hline Machaerium acutifolium Vogel & árv & $\mathrm{x}$ & & & & DS 1061 \\
\hline Mimosa bifurca Benth. & sub & & & $\mathrm{x}$ & $\mathrm{x}$ & DS 652 \\
\hline M. digitata Benth. & sub & & $\mathrm{x}$ & & & DS 901 \\
\hline M. distans Benth. & sub & $\mathrm{x}$ & $\mathrm{f}$ & & & DS 893 \\
\hline M. dolens Vell. ssp. acerba (Benth.) Barneby & sub & & $\mathrm{x}$ & & & DS 958 \\
\hline M. gracilis Benth. ssp. capillipes (Benth.) Barneby & erv & & $\mathrm{x}$ & & & DS 946 \\
\hline M. nuda Benth. var. nuda & sub & & $\mathrm{x}$ & & & DS 925 \\
\hline M. paucifolia Benth. & erv & & & & $\mathrm{x}$ & DS 1016 \\
\hline M. radula Benth. var. calycina (Benth.) Barneby & arb & & $\mathrm{f}$ & $\mathrm{x}$ & & DS 365 \\
\hline M. setosa Benth. subsp. setosa & arb & $\mathrm{x}$ & & & & DS 585 \\
\hline Plathymenia reticulata Benth. & árv & $\mathrm{x}$ & & & & DS 438 \\
\hline Poiretia latifolia Vogel & sub & $\mathrm{x}$ & & & & DS 968 \\
\hline Pterodon pubescens Benth. & árv & $\mathrm{x}$ & & & & DS 702 \\
\hline Senna pendula (Humb. \& Bonpl. ex Willd.) H.S. Irwin \& Barneby & arb & $\mathrm{x}$ & & & & DS 929 \\
\hline S. pilifera (Vogel) H.S. Irwin \& Barneby & sub & $\mathrm{x}$ & & & & DS 890 \\
\hline S. rugosa (G. Don) H.S. Irwin \& Barneby & arb & $\mathrm{x}$ & $\mathrm{x}$ & & & DS 408 \\
\hline Stryphnodendron adstringens (Mart.) Coville & árv & & $\mathrm{x}$ & & & DS 688 \\
\hline Stylosanthes guianensis var. gracilis (Kunth) Vogel & arb & $\mathrm{x}$ & $\mathrm{x}$ & & & DS 164 \\
\hline S. viscosa $\mathrm{Sw}$. & arb & & $\mathrm{f}$ & & $\mathrm{x}$ & DS 157 \\
\hline Tephrosia purpurea (L.) Pers. ssp. leptostachya (DC.) Burret & erv & $\mathrm{x}$ & $\mathrm{x}$ & & & DS 285 \\
\hline Vaiterea macrocarpa (Benth.) Ducke & árv & $\mathrm{x}$ & & & & DS 711 \\
\hline Vigna firmula (Benth.) Maréchal, Mascherpa \& Stainier & sub & & & & $\mathrm{f}$ & DS 530 \\
\hline V. peduncularis (Kunth) Fawcet \& Rendle & erv & $\mathrm{x}$ & & & & DS 970 \\
\hline Leguminosae sp. 1 & árv & & $\mathrm{x}$ & & & DS 177 \\
\hline \multicolumn{7}{|l|}{ LENTIBULARIACEAE } \\
\hline Genlisea filiformis A. St.-Hil. & erv & & $\mathrm{f}$ & $\mathrm{f}$ & & DS 256 \\
\hline Utricularia neottioides A. St.-Hil. \& Gérard & erv & & & $\mathrm{f}$ & & DS 244 \\
\hline U. simulans Pilger & erv & & & $\mathrm{x}$ & & DS 1028 \\
\hline U. subulata $\mathrm{L}$. & erv & & $\mathrm{f}$ & $\mathrm{x}$ & $\mathrm{x}$ & DS 1013 \\
\hline \multicolumn{7}{|l|}{ LOGANIACEAE } \\
\hline Strychnos bicolor Progel & arb & & & & $\mathrm{x}$ & DS 752 \\
\hline \multicolumn{7}{|l|}{ LORANTHACEAE } \\
\hline Struthanthus polyanthus Mart. & sub & $\mathrm{x}$ & & & & DS 1060 \\
\hline \multicolumn{7}{|l|}{ LYTHRACEAE } \\
\hline Cuphea linarioides Cham. \& Schltdl. & sub & & $\mathrm{f}$ & & & DS 774 \\
\hline C. lutescens Pohl ex Koehne & sub & $\mathrm{x}$ & & & & DS 403 \\
\hline C. micrantha Kunth & erv & & & & $\mathrm{x}$ & DS 992 \\
\hline C. thymoides Cham. \& Schltdl. & sub & $\mathrm{x}$ & $\mathrm{x}$ & & & DS 392 \\
\hline Cuphea sp. nov. & sub & & & $\mathrm{x}$ & $\mathrm{x}$ & DS 786 \\
\hline Diplusodon glocimarii T.B.Cavalc. & erv & & & $\mathrm{f}$ & & DS 234 \\
\hline D. ovatus Pohl & sub & & $\mathrm{x}$ & & & DS 879 \\
\hline D. villosissimus Pohl & sub & $\mathrm{x}$ & $\mathrm{x}$ & & & DS 306 \\
\hline D. virgatus Pohl & arb & $\mathrm{x}$ & $\mathrm{f}$ & & & DS 425 \\
\hline Lafoensia pacari A. St.-Hil. & árv & & $\mathrm{f}$ & & $\mathrm{x}$ & DS 532 \\
\hline \multicolumn{7}{|l|}{ MALPIGHIACEAE } \\
\hline Banisteriopsis campestris (A. Juss.) Little & arb & $\mathrm{x}$ & $\mathrm{f}$ & & & DS 772 \\
\hline B. laevifolia (A. Juss.) Gates & arb & $\mathrm{x}$ & & & & DS 983 \\
\hline B. pubipetala (A. Juss.) Cuatrec. & arb & $\mathrm{x}$ & & & & DS 677 \\
\hline B. stellaris (Griseb.) Gates & arb & $\mathrm{x}$ & $\mathrm{f}$ & $\mathrm{x}$ & $\mathrm{x}$ & DS 524 \\
\hline Byrsonima coccolobifolia Kunth & arb & & $\mathrm{x}$ & & & DS 1064 \\
\hline B. crassa Nied. & arb & & $\mathrm{x}$ & & & DS 449 \\
\hline
\end{tabular}


Tabela 2 (continuação)

\begin{tabular}{|c|c|c|c|c|c|c|}
\hline Famílias/Espécies & Hábito & 1 & 2 & 3 & 4 & Voucher \\
\hline Byrsonima crassifolia (L.) Kunth & árv & $\mathrm{x}$ & & & & DS 873 \\
\hline B. intermedia A. Juss. & arb & & $\mathrm{x}$ & & & DS 280 \\
\hline B. pachyphylla A. Juss. & árv & & $\mathrm{f}$ & & & DS 766 \\
\hline B. subterranea Brade \& Markgr. & sub & & & $\mathrm{x}$ & $\mathrm{x}$ & DS 563 \\
\hline B. verbascifolia (L.) DC. & árv & $\mathrm{x}$ & $\mathrm{f}$ & & & DS 595a \\
\hline Camarea affinis A. St.-Hil. & sub & & $\mathrm{x}$ & $\mathrm{x}$ & $\mathrm{x}$ & DS 885 \\
\hline C. ericoides A. St.-Hil. & erv & & $\mathrm{x}$ & & & DS 758 \\
\hline Galphimia brasiliensis A. Juss. & sub & $\mathrm{x}$ & & & & DS 437 \\
\hline Heteropterys campestris A. Juss. & arb & $\mathrm{x}$ & $\mathrm{x}$ & & & DS 405 \\
\hline H. tomentosa A. Juss. & arb & $\mathrm{x}$ & & $\mathrm{x}$ & & DS 500 \\
\hline Peixotoa reticulata Griseb. & arb & $\mathrm{x}$ & $\mathrm{f}$ & $x$ & $\mathrm{x}$ & DS 318 \\
\hline Pterandra pyroidea A. Juss. & sub & $\mathrm{x}$ & $\mathrm{x}$ & & & DS 683 \\
\hline Tetrapterys microphylla (A. Juss.) Nied. & arb & & & $\mathrm{x}$ & $\mathrm{x}$ & DS 620 \\
\hline Tetrapterys sp. & arb & $\mathrm{x}$ & $\mathrm{x}$ & & & DS 687 \\
\hline \multicolumn{7}{|l|}{ MALVACEAE } \\
\hline Byttneria sagitifolia A. St-Hil. & erv & $\mathrm{x}$ & $\mathrm{f}$ & & & DS 419 \\
\hline Helicteres sacarolha A. St.-Hil., A. Juss. \& Cambess. & sub & $\mathrm{x}$ & $\mathrm{f}$ & & & DS 439 \\
\hline Krapovickasia macrodon (DC.) Fryxell & erv & & $\mathrm{x}$ & & & DS 842 \\
\hline Luehea candicans Mart. & árv & $\mathrm{f}$ & & & & DS 829 \\
\hline L. divaricata Mart. & árv & $\mathrm{x}$ & & & & DS 672 \\
\hline L. grandiflora Mart. & árv & $\mathrm{x}$ & & & & DS 502 \\
\hline Melochia pilosa (Mill.) Fawc. \& Rendle & sub & $\mathrm{x}$ & & & & DS 428 \\
\hline Peltaea edouardii (Hochr.) Krapov. \& Cristóbal & erv & $\mathrm{x}$ & & & & DS 896 \\
\hline Peltaea sp. 1 & sub & & $\mathrm{x}$ & & & DS 832 \\
\hline Peltaea sp. 2 & sub & & $\mathrm{x}$ & & & DS 947 \\
\hline Pseudobombax marginatum (A. St.-Hil.) A. Robyns & árv & $\mathrm{x}$ & & & & DS 985 \\
\hline Sida cerradoensis Krapov. & erv & & $\mathrm{f}$ & & & DS 154 \\
\hline S. linifolia Cav. & erv & & $\mathrm{x}$ & & $\mathrm{x}$ & DS 957 \\
\hline \multicolumn{7}{|l|}{ MELASTOMATACEAE } \\
\hline Acisanthera alsinifolia (DC.) Triana & erv & & $\mathrm{f}$ & & & DS 1024 \\
\hline Cambessedesia hilariana (Kunth) DC. & erv & $\mathrm{x}$ & & $\mathrm{x}$ & $\mathrm{x}$ & DS 359 \\
\hline C. regnelliana Cogn. & erv & & & $\mathrm{f}$ & $\mathrm{x}$ & DS 323 \\
\hline Chaetostoma pungens (Spreng.) Cogn. & sub & & & $\mathrm{x}$ & $\mathrm{f}$ & DS 349 \\
\hline Clidemia sericea Don & arb & & & $\mathrm{x}$ & $\mathrm{x}$ & DS 1000 \\
\hline Leandra polystachya (Naudin) Cogn. & sub & $\mathrm{x}$ & & & & DS 709 \\
\hline Macairea radula (Bonpl.) DC. & arb & $\mathrm{x}$ & $\mathrm{f}$ & & & DS 684 \\
\hline Miconia albicans (Sw.) Triana & arb & $\mathrm{x}$ & $\mathrm{f}$ & $\mathrm{x}$ & & DS 669 \\
\hline M. fallax DC. & arb & & & $\mathrm{x}$ & & DS 732 \\
\hline M. ferruginata DC. & árv & & & $\mathrm{x}$ & $\mathrm{x}$ & DS 376 \\
\hline M. rubiginosa (Bonpl.) DC. & arb & & & $\mathrm{x}$ & & DS 486 \\
\hline Microlicia cuneata Naudin & sub & & & $\mathrm{x}$ & & DS 916 \\
\hline M. euphorbioides Mart. & sub & $\mathrm{x}$ & & & & DS 418 \\
\hline M. fasciculata Mart. ex Naud. & sub & & $\mathrm{f}$ & & & DS 1025 \\
\hline M. polystemma Naud. & sub & & $\mathrm{f}$ & $\mathrm{f}$ & & DS 252 \\
\hline Pterolepis perpusilla (Naud.) Cogn. & erv & & & & $\mathrm{f}$ & DS 144 \\
\hline P. repanda (DC.) Triana & sub & & $\mathrm{x}$ & & & DS 944 \\
\hline Siphanthera cordata Pohl ex DC. & erv & & $\mathrm{f}$ & & & DS 1026 \\
\hline S. dawsonii Wurdack & erv & & & & $\mathrm{x}$ & DS 989 \\
\hline Svitramia sp. nov. & sub & $\mathrm{x}$ & & $\mathrm{x}$ & $\mathrm{x}$ & DS 474 \\
\hline Tibouchina aegopogon Cogn. & arb & & & $\mathrm{x}$ & & DS 918 \\
\hline T. heteromalla (D.Don) Cogn. & arb & $\mathrm{x}$ & & $\mathrm{f}$ & $\mathrm{x}$ & DS 331 \\
\hline \multicolumn{7}{|l|}{ MENISPERMACEAE } \\
\hline Cissampelos ovalifolia DC. & erv & $\mathrm{x}$ & & & & DS 860 \\
\hline \multicolumn{7}{|l|}{ MORACEAE } \\
\hline Brosimum gaudichaudii Trécul & arb & & $\mathrm{x}$ & & & DS 838 \\
\hline \multicolumn{7}{|l|}{ MYRISTICACEAE } \\
\hline Virola sebifera Aubl. & árv & $\mathrm{f}$ & & & & DS 943 \\
\hline
\end{tabular}


Tabela 2 (continuação)

\begin{tabular}{|c|c|c|c|c|c|c|}
\hline Famílias/Espécies & Hábito & 1 & 2 & 3 & 4 & Voucher \\
\hline \multicolumn{7}{|l|}{ MYRSINACEAE } \\
\hline Myrsine guianensis (Aubl.) Kuntze & árv & $\mathrm{x}$ & $\mathrm{x}$ & $\mathrm{x}$ & $\mathrm{x}$ & DS 760 \\
\hline \multicolumn{7}{|l|}{ MYRTACEAE } \\
\hline Campomanesia pubescens (DC.) O. Berg & arb & & $\mathrm{x}$ & & & DS 1052 \\
\hline Eugenia livida O. Berg & arb & $\mathrm{x}$ & & & & DS 592 \\
\hline E. punicifolia (Kunth) DC. & arb & & $\mathrm{x}$ & & & DS 571 \\
\hline Myrcia fallax (Rich.) DC. & arb & $\mathrm{x}$ & & & & DS 827 \\
\hline M. liguiformis (O. Berg) N. Silveira & arb & & & $\mathrm{x}$ & $\mathrm{x}$ & DS 658 \\
\hline M. rhodeosepala Kiaersk. & arb & & $\mathrm{x}$ & & & DS 692 \\
\hline M. torta DC. & arb & & & $\mathrm{x}$ & & DS 372 \\
\hline M. uberavensis O. Berg & arb & $\mathrm{x}$ & $\mathrm{x}$ & $\mathrm{x}$ & $\mathrm{x}$ & DS 647 \\
\hline M. variabilis DC. & arb & $\mathrm{x}$ & & & & DS 891A \\
\hline Psidium firmum $\mathrm{O}$. Berg & arb & & $\mathrm{x}$ & & & DS 1053 \\
\hline$P$. grandifolium DC. & arb & & $\mathrm{x}$ & & & DS 1055 \\
\hline P. multiflorum Cambess. & arb & $\mathrm{x}$ & & & & DS 857 \\
\hline \multicolumn{7}{|l|}{ NYCTAGINACEAE } \\
\hline Neea theifera Oerst. & árv & $\mathrm{x}$ & $\mathrm{x}$ & $\mathrm{x}$ & $\mathrm{x}$ & DS 710 \\
\hline Ouratea hexasperma (A. St.-Hil.) Baill. & árv & & & $\mathrm{x}$ & $\mathrm{x}$ & DS 659 \\
\hline O. spectabilis (Mart. ex Engl.) Engl. & árv & $\mathrm{x}$ & $\mathrm{x}$ & $\mathrm{x}$ & $\mathrm{x}$ & DS 694 \\
\hline Sauvagesia racemosa A. St.-Hil. & sub & & $\mathrm{f}$ & & & DS 165 \\
\hline \multicolumn{7}{|l|}{ ORCHIDACEAE } \\
\hline Cyrtopodium eugenii Rchb.f. & erv & & & $\mathrm{x}$ & & DS 547 \\
\hline Epistephium sclerophyllum Lindl. & erv & & $\mathrm{x}$ & & & DS 301 \\
\hline Galeandra montana Barb. Rodr. & erv & $\mathrm{x}$ & & & & DS 933 \\
\hline Habenaria glazioviana Krzl. & erv & & $\mathrm{f}$ & & & DS 1023 \\
\hline H. hamata Barb. Rodr. & erv & $\mathrm{x}$ & & & & DS 424 \\
\hline H. leprieuri Rchb.f. & erv & & & $\mathrm{x}$ & & DS 887 \\
\hline Sacoila lanceolata (Aubl.) Garay & erv & & & & $\mathrm{x}$ & DS 660 \\
\hline \multicolumn{7}{|l|}{ OROBANCHACEAE } \\
\hline Buchnera lavandulacea Cham. \& Schltdl. & erv & $\mathrm{x}$ & & $\mathrm{f}$ & & DS 420 \\
\hline B. rosea Kunth & erv & & & & $\mathrm{x}$ & DS 1033 \\
\hline \multicolumn{7}{|l|}{ OXALIDACEAE } \\
\hline Oxalis hirsutissima Mart. ex Zucc. & sub & $\mathrm{x}$ & $\mathrm{x}$ & $\mathrm{x}$ & $\mathrm{x}$ & DS 304 \\
\hline O. physocalyx Zucc. ex Prog. & sub & $\mathrm{x}$ & & & & DS 448 \\
\hline \multicolumn{7}{|l|}{ PALMAEE } \\
\hline Syagrus flexuosa (Mart.) Becc. & arb & $\mathrm{x}$ & & & & DS 446 \\
\hline \multicolumn{7}{|l|}{ PASSIFLORACEAE } \\
\hline Passiflora clathrata Mast. & erv & $\mathrm{x}$ & & & & DS 858 \\
\hline \multicolumn{7}{|l|}{ PHYLLANTHACEAE } \\
\hline Phyllanthus orbiculatus L.C. Rich. & sub & $\mathrm{x}$ & & & & DS 432 \\
\hline Phyllanthus sp. & erv & & & & $\mathrm{x}$ & DS 990 \\
\hline \multicolumn{7}{|l|}{ POLYGALACEAE } \\
\hline Polygala cneorum A. St.-Hil. & erv & & & $\mathrm{x}$ & & DS 548 \\
\hline P. dusenii Norl. & erv & & & $\mathrm{x}$ & $\mathrm{x}$ & DS 668 \\
\hline P. hebeclada DC. & erv & $\mathrm{x}$ & & & & DS 421 \\
\hline P. hirsuta A. St.-Hil. & erv & & & & $\mathrm{x}$ & DS s.n. \\
\hline P. longicaulis Kunth & erv & $\mathrm{f}$ & $\mathrm{x}$ & & $\mathrm{x}$ & DS 286 \\
\hline P. minima Pohl ex A.W. Benn. & erv & & $\mathrm{x}$ & & $\mathrm{x}$ & DS 1001 \\
\hline P. molluginifolia A. St.-Hil. \& Moq. & sub & $\mathrm{x}$ & $\mathrm{x}$ & & & DS 291 \\
\hline P. nudicaulis A.W. Benn. & erv & & $\mathrm{x}$ & $\mathrm{x}$ & & DS 288 \\
\hline P. tamariscea Mart. & erv & $\mathrm{x}$ & & & & DS 1062 \\
\hline \multicolumn{7}{|l|}{ PROTEACEAE } \\
\hline Roupala montana Aubl. & arb-árv & $\mathrm{x}$ & $\mathrm{x}$ & & $\mathrm{x}$ & DS 639 \\
\hline \multicolumn{7}{|l|}{ RHAMNACEAE } \\
\hline Crumenaria choretroides Mart. ex Reissek & erv & $\mathrm{x}$ & & & & DS 809 \\
\hline \multicolumn{7}{|l|}{ RUBIACEAE } \\
\hline Borreria poaya (A. St.-Hil.) DC. & erv & $\mathrm{x}$ & $\mathrm{x}$ & & & DS 463 \\
\hline B. aff. poaya (A. St.-Hil.) DC. & erv & & & $\mathrm{f}$ & & DS 549 \\
\hline
\end{tabular}


Tabela 2 (continuação)

\begin{tabular}{|c|c|c|c|c|c|c|}
\hline Famílias/Espécies & Hábito & 1 & 2 & 3 & 4 & Voucher \\
\hline Borreria suaveolens G. Мey. & sub & & & $\mathrm{x}$ & & DS 356 \\
\hline B. tenella Cham. \& Schltdl. & erv & & $\mathrm{x}$ & & & DS 292 \\
\hline B. warmingii K. Schum. & erv & $\mathrm{x}$ & $\mathrm{f}$ & & & DS 382 \\
\hline Coccocypselum lanceolatum (Ruiz \& Pav.) Pers. & erv & $\mathrm{x}$ & & & & DS 429 \\
\hline Declieuxia fruticosa (Willd. ex Roem. \& Schult.) Kuntze & sub & $\mathrm{x}$ & $\mathrm{x}$ & $\mathrm{x}$ & $\mathrm{x}$ & DS 329 \\
\hline Galianthe grandifolia E.L. Cabral & sub & $\mathrm{x}$ & $\mathrm{x}$ & & & DS 303 \\
\hline G. liliifolia (Standl.) E.L. Cabral & sub & $\mathrm{x}$ & & & & DS 932 \\
\hline Guettarda viburnoides Cham. \& Schltdl. & árv & & $\mathrm{x}$ & & & DS 836 \\
\hline Mitracarpus frigidus K. Schum. & erv & & $\mathrm{x}$ & & & DS 294 \\
\hline Palicourea coriacea (Cham.) K. Schum. & arb & & $\mathrm{f}$ & & & DS 755 \\
\hline P. rigida Kunth & arb & $\mathrm{x}$ & $\mathrm{x}$ & $\mathrm{x}$ & $\mathrm{x}$ & DS 761 \\
\hline Rudgea viburnoides (Cham.) Benth. & árv & $\mathrm{x}$ & & & & DS 673 \\
\hline Sabicea brasiliensis Wernh. & arb & $\mathrm{x}$ & $\mathrm{f}$ & $\mathrm{x}$ & $\mathrm{x}$ & DS 320 \\
\hline Tocoyena formosa (Cham. \& Schltdl.) K. Schum. & árv & $\mathrm{f}$ & & & $\mathrm{x}$ & DS 850 \\
\hline \multicolumn{7}{|l|}{ SALICACEAE } \\
\hline Casearia sylvestris $\mathrm{Sw}$. & arb & $\mathrm{x}$ & $\mathrm{x}$ & & $\mathrm{x}$ & DS 654 \\
\hline \multicolumn{7}{|l|}{ SAPINDACEAE } \\
\hline Sapindaceae sp. & sub & $\mathrm{x}$ & & & & DS 974 \\
\hline Smilax goyazana A. DC. & sub & & $\mathrm{x}$ & & & DS 1050 \\
\hline S. polyantha Griseb. & sub & & & $\mathrm{x}$ & $\mathrm{f}$ & DS 751 \\
\hline \multicolumn{7}{|l|}{ SOLANACEAE } \\
\hline Cestrum pedicellatum Sendtn. & arb & & $\mathrm{x}$ & & & DS 645 \\
\hline Solanum lycocarpum A. St.-Hil. & árv & & $\mathrm{f}$ & & & DS 92 \\
\hline \multicolumn{7}{|l|}{ STYRACACEAE } \\
\hline Styrax camporum Pohl & arb & & & $\mathrm{f}$ & $\mathrm{x}$ & DS 477 \\
\hline \multicolumn{7}{|l|}{ TURNERACEAE } \\
\hline Piriqueta aurea (Cambess.) Urb. & arb & $\mathrm{x}$ & & & & DS 804 \\
\hline P. cistoides (L.) Griseb. & erv & & & & $\mathrm{x}$ & DS 1004 \\
\hline Turnera lanceolata Cambess. & erv & & & $\mathrm{x}$ & & DS s.n. \\
\hline T. oblongifolia Cambess. & sub & $\mathrm{x}$ & & $\mathrm{x}$ & & DS 697 \\
\hline \multicolumn{7}{|l|}{ UMBELLIFERAE } \\
\hline Eryngium juncifolium (Urb.) Mathias \& Constance & erv & $\mathrm{x}$ & $\mathrm{x}$ & & & DS 386 \\
\hline E. paniculatum Cav. \& Dombey ex F. Delaroche & erv & & $\mathrm{x}$ & & & DS 102 \\
\hline Eryngium sp. 1 & erv & $\mathrm{x}$ & & & & DS 892 \\
\hline Eryngium sp. 2 & erv & & $\mathrm{x}$ & & & DS 831 \\
\hline \multicolumn{7}{|l|}{ VELLOZIACEAE } \\
\hline Vellozia glauca Pohl & arb & $\mathrm{x}$ & $\mathrm{x}$ & $\mathrm{x}$ & $\mathrm{x}$ & DS 665 \\
\hline V. obtecta Mello-Silva & arb & & & $\mathrm{x}$ & & DS 919 \\
\hline V. peripherica Mello-Silva & arb & $\mathrm{x}$ & & $\mathrm{x}$ & & DS 690 \\
\hline \multicolumn{7}{|l|}{ VERBENACEAE } \\
\hline Lantana glaziovii Moldenke & erv & & $\mathrm{x}$ & & & DS 763 \\
\hline Lippia lasiocalycina Cham. & arb & $\mathrm{x}$ & & & & DS 705 \\
\hline L. lupulina Cham. & sub & $\mathrm{x}$ & & & $\mathrm{x}$ & DS 663 \\
\hline L. sericea Benth. & sub & & $\mathrm{x}$ & & & DS 289 \\
\hline L. stachyoides Cham. & sub & & $\mathrm{f}$ & & & DS 151 \\
\hline L. vernonioides Cham. & erv & $\mathrm{x}$ & & & & DS 513 \\
\hline Stachytarpheta gesnenerioides Cham. & sub & $\mathrm{x}$ & & & & DS 387 \\
\hline $\begin{array}{l}\text { S. longispicata (Pohl) S. Atkins ssp. brevibracteata } \\
\text { (Moldenke) S. Atkins }\end{array}$ & arb & & & & $\mathrm{f}$ & DS 534 \\
\hline \multicolumn{7}{|l|}{ VITACEAE } \\
\hline Cissus erosa Rich. & erv & & $\mathrm{f}$ & & $\mathrm{x}$ & DS 113 \\
\hline \multicolumn{7}{|l|}{ VOCHYSIACEAE } \\
\hline Qualea grandiflora Mart. & árv & & $\mathrm{x}$ & & & DS 576 \\
\hline Q. multiflora Mart. & árv & $\mathrm{x}$ & $\mathrm{f}$ & $\mathrm{x}$ & $\mathrm{x}$ & DS 851 \\
\hline Q. parviflora Mart. & árv & & $\mathrm{x}$ & $\mathrm{f}$ & $\mathrm{x}$ & DS 232 \\
\hline Salvertia convallariodora A. St.-Hil. & árv & & & $\mathrm{f}$ & & DS 544a \\
\hline
\end{tabular}


Tabela 2 (continuação)

\begin{tabular}{|c|c|c|c|c|c|c|}
\hline Famílias/Espécies & Hábito & 1 & 2 & 3 & 4 & Voucher \\
\hline Vochysia cinnamomea Pohl & árv & & $\mathrm{f}$ & & $\mathrm{X}$ & DS 325 \\
\hline V. rufa Mart. & árv & $\mathrm{x}$ & & & & DS 416 \\
\hline V. sessilifolia Warm. & arb & & & $\mathrm{x}$ & $\mathrm{f}$ & DS 366 \\
\hline \multicolumn{7}{|l|}{ XYRIDACEAE } \\
\hline Xyris asperula Mart. & erv & & $\mathrm{f}$ & $\mathrm{f}$ & $\mathrm{x}$ & DS 327 \\
\hline X. seubertii L.A. Nilsson & erv & & & $\mathrm{x}$ & & DS 360 \\
\hline X. tenella Kunth & erv & & & $\mathrm{x}$ & & DS 1030 \\
\hline$X$. tortula Mart. & erv & $\mathrm{x}$ & & $\mathrm{x}$ & & DS 400 \\
\hline
\end{tabular}

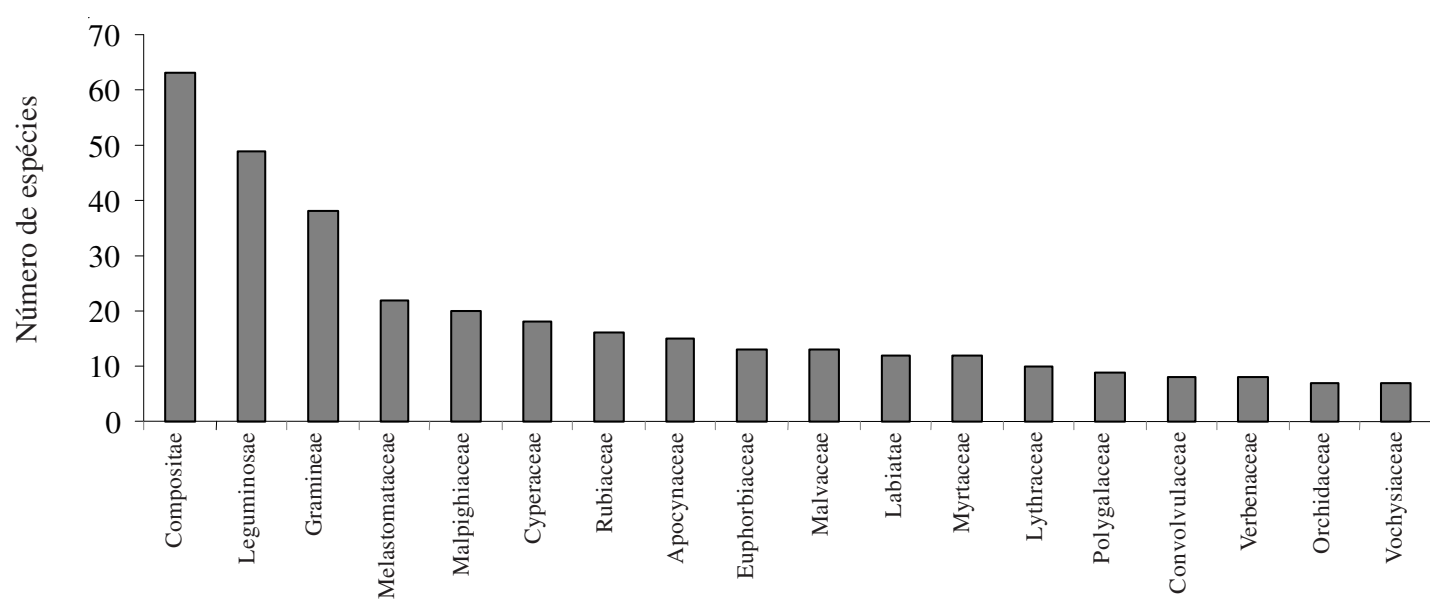

Figura 2. Principais famílias amostradas em Pedregulho, SP, Brasil, ordenadas por número de espécies.

entre as áreas situadas no P.E. das Furnas do Bom Jesus, e 68 espécies entre as áreas em Estreito. Somente 20 espécies são comuns às quatro áreas.

A análise por UPGMA agrupa as áreas de Estreito, com um alto valor de similaridade $(\mathrm{S}=0,55)$, contra as áreas do P.E. Furnas do Bom Jesus, com valor de similaridade inferior ( $\mathrm{S}=0,40)$ (Fig. 3). Os valores de similaridades foram mais baixos entre as áreas 2 e 3 $(\mathrm{S}=0,24)$, áreas 1 e $3(\mathrm{~S}=0,25)$, áreas 2 e 4, áreas 1 e $4(\mathrm{~S}=0,27)$, e entre o P.E. Furnas do Bom Jesus e o distrito de Estreito $(S=0,25)$.

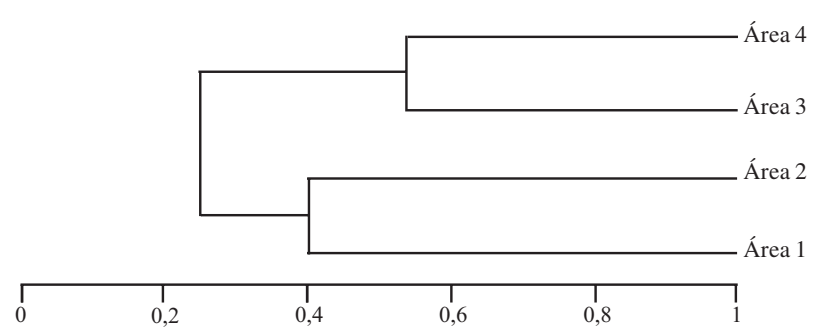

Figura 3. Dendrograma obtido pela análise por UPGMA das quatro parcelas amostradas e suas adjacências, utilizando-se o coeficiente de similaridade de Sorensen.
A maioria das espécies deste estudo apresenta padrões de distribuição amplos. Oito deles englobam as espécies cuja distribuição em São Paulo restringese a Pedregulho, ou eventualmente a Pedregulho e a Rifaina, município vizinho e com características geomorfológicas semelhantes (Almeida et al. 1981).

\section{Endêmica de Pedregulho}

Padrão representado somente por Cuphea sp., possivelmente inédita, do cerrado e campo rupestre (Cavalcanti \& Graham 2002).

\section{Pedregulho e Serra da Canastra}

Svitramia sp. nov. (Romero \& Martins 2002), Stevia hilarii e Vernonia sp. nov. (Nakajima \& Semir 2001), citadas como endêmicas da Serra da Canastra, ocorrem também em Pedregulho, sobre afloramentos quartzíticos ou areníticos.

\section{Pedregulho e sudoeste de Minas Gerais}

É um padrão de distribuição em “U”, periférico à Cadeia do Espinhaço (Romero 2002), de espécies 
exclusivas das porções sudoeste e sul dos campos rupestres de Minas Gerais. Diplusodon glocimarii, Eremanthus seidelii, Vellozia obtecta e $V$. peripherica confirmam esse padrão, estendendose, porém, até Pedregulho.

Diplusodon glocimarii era considerado endêmico da Serra da Canastra e Capitólio (Cavalcanti 2004).

\section{Pedregulho e Minas Gerais}

Calea graminifolia segue o padrão anterior, mas ocorre também na porção mineira da Cadeia do Espinhaço.

5. Pedregulho, sul e sudoeste de Minas Gerais e Goiás

Este padrão é baseado naquele de espécies exclusivas das serras de Goiás e do sudoeste de Minas Gerais (Romero 2002). A distribuição de Chresta scapigera segue este padrão, porém se estende até Pedregulho e o sul de Minas Gerais, em fisionomias mais abertas de cerrado e em campo rupestre.

6. Pedregulho, Minas Gerais, Centro-Oeste e países limítrofes

Padrão comum a muitas espécies (ou variedade, no caso de Tibouchina aegopogon) que se distribuem pelas serras do Centro-Oeste, sudoeste mineiro, Pedregulho, Planalto Sul de Minas e alcança a porção mineira da Cadeia do Espinhaço, podendo se estender até a Bolívia. Ocorrem em campo rupestre e cerrado, algumas em campo limpo, campo sujo e afloramento rochoso. As espécies amostradas em Pedregulho que apresentam esse padrão são Barjonia laxa (GO, MG, MT, SP), Camarea ericoides (GO, MG, MS, MT, SP), Chamaecrista ochnacea (MG, MT, SP), Deianira pallescens (DF, GO, MG, MT, SP), Diplusodon ovatus (GO, MG, MS, MT, SP), Diplusodon villosissimus (MG, MT, SP), Hyptidendron canum (DF, GO, MG, MS, MT, SP, Bolívia), Lychnophora ericoides (DF, GO, MG, SP), Manihot triphylla (DF, GO, MG, SP), Mimosa digitata (DF, GO, MG, SP), Polygala nudicaulis (GO, MG, SP), Smilax goyazana (DF, GO, MG, MS, MT, SP, Bolívia), Stachytarpheta longispicata (DF, GO, MG, SP), Strophopappus speciosus (DF, GO, MG, MS, MT, SP), Tetrapterys microphylla (GO, MG, SP), Tibouchina aegopogon var. aegopogon (DF, GO, MG, SP), Vochysia sessilifolia (GO, MG, MT, SP) e Wedelia puberula (GO, MG, SP).

\section{Pedregulho, Minas Gerais, Centro-Oeste e Bahia}

Padrão semelhante ao anterior, mas que engloba também a porção baiana da Cadeia do Espinhaço. As espécies coletadas em Pedregulho que apresentam esse padrão são: Myrcia torta (BA, DF, MG, GO, SP), Siphanthera cordata (BA, DF, GO, MG, MT, SP), Vellozia glauca (BA, GO, MG, SP), Vernonia buddleiifolia (BA, GO, MG, MS, MT, SP), Vernonia schwenkiifolia (BA, GO, MG, SP) e Wunderlichia mirabilis (BA, GO, MG, SP).

8. Pedregulho como limite sul de distribuições neotropicais amplas

Espécies amplamente distribuídas nas Américas Central e do Sul, cujo limite meridional são os Estados de Minas Gerais e o de São Paulo, sendo que neste último são restritas à região de Pedregulho. Ocorrem geralmente em ambientes abertos como cerrado e campo rupestre, mais raramente em florestas. Apresentam esse padrão: Habenaria leprieuri (GO, MG, PA, RO, SP, Bolívia, Trinidad e Tobago, Venezuela), Mesosetum loliiforme (AM, AP, BA, CE, DF, GO, MA, MG, MS, MT, PA, PI, RN, RO, SP, Colômbia, Cuba, Guianas, Guiana Francesa, Suriname, Venezuela), Siphanthera dawsonii (GO, MG, MT, PA, SP, Venezuela), Trimezia lutea (DF, GO, PI, RJ, SP, TO, Colômbia, Venezuela), Vochysia rufa (BA, DF, GO, MA, MG, MS, MT, PA, PI, RO, SP, Bolívia).

Das 39 espécies aqui analisadas, 17 foram encontradas somente em Estreito, 16 somente no P.E. das Furnas do Bom Jesus e seis em ambas regiões. Os ambientes de ocorrência são os mais variados, mas somente Calea graminifolia foi encontrada apenas em campo rupestre, e Diplusodon ovatus, Habenaria leprieuri, Mimosa digitata e Polygala nudicaulis não foram encontradas em campo rupestre. Sobre as demais especies, as informações disponíveis revelam que ocorrem em ambos os ambientes.

\section{Discussão}

Os cerrados paulistas são peculiares, pois, juntamente com os do Paraná, constituem o limite sul de ocorrência dessa vegetação e estão sujeitos a geadas e a períodos de seca mais curtos do que os cerrados do Planalto Central (Durigan et al. 2004). O município de Pedregulho, ainda, está situado em uma região distintiva, onde coincidem transições geomorfológica e climática. Essa região, como a maior parte de Minas Gerais, apresenta um período de seca de 4-5 meses, enquanto que nas demais áreas de cerrado paulista o clima é úmido com um período de seca mais curto, de 1-3 meses (Nimer 1989). A importância da influência do clima nos padrões de 
similaridade florística do cerrado tem sido indicada por outros estudos (Castro \& Martins 1999; Ratter et al. 1996; 2003; Durigan et al. 2003a).

A maioria das espécies da listagem florística de Pedregulho apresenta ampla distribuição. Uma fração menor é, no Estado de São Paulo, restrita a essa região e pode se distribuir nos estados ao norte, e também em áreas mais ao sul em Minas Gerais e Mato Grosso do Sul. Uma característica comum a quase todas elas é sua ocorrência em campos rupestres, embora também ocorram em outras fisionomias, geralmente abertas. Apesar de predominantes na Cadeia do Espinhaço (Giulietti \& Pirani 1988; Giulietti et al. 1997), os campos rupestres também ocorrem em serras do sul e sudoeste de Minas Gerais, de Goiás e do Distrito Federal (Giulietti et al. 2000), vinculadas ao Planalto Sul de Minas e ao Maciço Goiano, ligados pelo Arco da Canastra, modelados em rochas pré-cambrianas (Moreira 1977; Moreira \& Camelier 1977).

$\mathrm{Na}$ Cadeia do Espinhaço, os padrões restritos, como endemismos locais e disjunções com a restinga e com serras de Goiás, são os mais comuns. Entretanto existem padrões mais amplos, como os de distribuição pela América tropical, principalmente em campos e cerrados, e as disjunções com o Planalto das Guianas (Giulietti \& Pirani 1988). Poucos estudos versam sobre padrões de distribuição geográfica nos campos rupestres do cinturão orogênico das serras de Goiás e Minas Gerais, mas dois padrões são reconhecidos: um de espécies endêmicas do sul e sudoeste de Minas Gerais, e outro de espécies que ocorrem nas serras de Goiás e no sudoeste mineiro (Romero 2002). Com o presente estudo, esses padrões se ampliam para algumas espécies, passando a incluir a região de Pedregulho.

A proximidade geográfica e a similaridade geológica entre o extremo nordeste de São Paulo e os Planaltos da Canastra são fatores que influenciam a composição florística diferenciada da região de Pedregulho. A fisionomia de Estreito é característica da região da Canastra e das represas de Furnas no sul mineiro, onde se notam as seqüências de quartzitos plaqueados, pertencentes a uma formação do grupo Canastra (Machado-Filho et al. 1983), diferindo-se do cerrado arenoso do P.E. das Furnas do Bom Jesus. A fisionomia da vegetação de Estreito é semelhante à de Delfinópolis, considerada como campo rupestre por Bonifácio-Silva (dados não publicados). A definição de campo rupestre não é clara (Giulietti et al. 1987) e pode ser discordante em relação às diferentes fisionomias, substratos e localização geográfica
(Giulietti \& Pirani 1988; Harley \& Simmons 1986; Ribeiro \& Walter 1998; Vitta 2002). A falta de clareza do termo também leva a dificuldades em definir padrões de distribuição de suas espécies e, dependendo do conceito adotado, mais ou menos espécies seriam consideradas exclusivas do campo rupestre, e que também provavelmente podem ocorrer em fisionomias adjuntas e ecótonos entre elas.

Ainda que um melhor posicionamento da flora de Pedregulho no âmbito das fisionomias do cerrado requeira outros estudos, a região abriga populações de espécies únicas no Estado de São Paulo. Este estudo confirma a singularidade florística dessa região e enfatiza a importância da preservação das áreas marginais do rio Grande, carentes de áreas de proteção.

\section{Agradecimentos}

Ao Conselho Nacional de Desenvolvimento Científico e Tecnológico (CNPq), pela bolsa concedida.

\section{Referências bibliográficas}

Almeida, F.F.M. 1964. Fundamentos geológicos do relevo paulista. Boletim do Instituto Geográfico e Geológico 41: 169-263.

Almeida, F.F.M.; Hasuy, Y.; Ponçano, W.L.; Dantas, A.S.L.; Carneiro, C.D.R.; Melo, M.S. \& Bistrichi, C.A. 1981. Mapa geológico do Estado de São Paulo: escala 1:500.000 volumes I e II. São Paulo, Instituto de Pesquisa Tecnológica do Estado de São Paulo, Divisão de Minas e Geologia Aplicada.

Angiosperm Phylogeny Group. 2003. An update of the Angiosperm Phylogeny Group classification for the orders and families of flowering plants: APG II. Botanical Journal of Linnean Society 141: 399-436.

Barbosa, L.M. \& Nunes, J.A. (coords.). 2001. Atlas das unidades de conservação do Estado de São Paulo, parte II: interior. São Paulo, Secretaria Estadual de Meio Ambiente. Metalivros.

Bonifácio-Silva, A.C. 2001. Levantamento florístico de cinco áreas em Delfinópolis, Minas Gerais - Brasil. Dissertação de Mestrado. Ribeirão Preto, Universidade de São Paulo.

Branco, I.C.; Domingues, E.N.; Serio, F.C.; Del Cali, I.H.; Mattos, I.A.; Bertoni, J.A.; Rossi, M.; Eston, M.R.; Pfeifer, R.M. \& Andrade, W.J. 1991. Plano de manejo - Parque Estadual das Furnas do Bom Jesus, município de Pedregulho, SP. Revista do Instituto Florestal 3: 137-155.

Brito, M.C.W. (coord.). 1997. Cerrado: Bases para conservação e uso sustentável das áreas de cerrado do Estado de São Paulo. São Paulo, Série PROBIO/SP, Secretaria do Meio Ambiente.

Castro, A.A.J.F. \& Martins, F.R. 1999. Cerrados do Brasil e do Nordeste: caracterização, área de ocupação e considerações sobre a sua fitodiversidade. Pesquisa em foco, São Luís 7: 147-178. 
Cavalcanti, T.B. 2004. Novos táxons, novos status, novo sinônimo e lectotipificações em Diplusodon Pohl (Lythraceae). Boletim de Botânica da Universidade de São Paulo 22: 1-14.

Cavalcanti, T.C. \& Graham, S.G. 2002. Lythraceae. Pp. 163-180. In: M.G.L. Wanderley; G.J. Shepherd \& A.M. Giulietti (coords.). Flora fanerogâmica do Estado de São Paulo. v.2. São Paulo, Fapesp, Hucitec.

Durigan, G.; Franco, G.A.D.C. \& Siqueira, M.F. 2004. A vegetação dos remanescentes de cerrado no Estado de São Paulo. Pp. 29-56. In: M.D. Bitencourt \& R.R. Mendonça (orgs.). Viabilidade de conservação dos remanescentes de cerrado do Estado de São Paulo. São Paulo, Annablume, Fapesp.

Durigan, G.; Ratter, J.A.; Bridgewater, S.; Siqueira, M.F. \& Franco, G.A.D.C. 2003a. Padrões fitogeográficos do cerrado paulista sob uma perspectiva regional. Hoehnea 30: 39-51.

Durigan, G.; Siqueira, M.F. \& Franco, G.A.D.C. 2002. A vegetação de cerrado no Estado de São Paulo. Pp. 53-54. In: E.L. Araújo (ed.). Biodiversidade, Conservação e Uso Sustentável da Flora do Brasil. Recife, Universidade Federal Rural de Pernambuco, Sociedade Botânica do Brasil.

Durigan, G.; Siqueira, M.F.; Franco, G.A.D.C.; Bridgewater, S. \& Ratter, J.A. 2003b. The vegetation of priority areas for cerrado conservation in São Paulo state, Brazil. Edinburgh Journal of Botany 60: 217-241.

Gatto, L.C.S.; Ramos, V.L.S.; Nunes, B.T.A.; Mamede, L.; Góes, M.H.B.; Mauro, C.A.; Alvarenga, S.M.; Franco, E.M.S.; Quirico, A.F. \& Neves, L.B. 1983. Geomorfologia. Pp. 305-384. In: Projeto RadamBrasil. Levantamento de Recursos Naturais. v. 32. Rio de Janeiro, Ministério de Energia e Minas, Secretaria Geral.

Giulietti, A.M.; Menezes, N.L.; Pirani, J.R.; Meguro, M. \& Wanderley, M.G.L. 1987. Flora da Serra do Cipó, Minas Gerais: caracterização e lista das espécies. Boletim de Botânica da Universidade de São Paulo 9: 1-151.

Giulietti, A.M. \& Pirani, J.R. 1988. Patterns of geographic distribution of some plant species from the Espinhaço Range, Minas Gerais and Bahia, Brazil. Pp. 39-69. In: P.E. Vanzolini \& W.R. Heyer (eds.). Proceedings of a workshop on Neotropical Distribution Patterns. Rio de Janeiro, Academia Brasileira de Ciências.

Giulietti, A.M.; Pirani, J.R. \& Harley, R.M. 1997. Espinhaço Range Region, Eastern Brazil. Pp. 397-404. In: S.D. Davis; V.H. Heywood; O. Herrera-MacBryde; J. Villa-Lobos \& A.C. Hamilton (eds.). Centres of Plant Diversity. Cambridge, The World Wide Fund for Nature (WWF), The World Conservation Union (IUCN).

Giulietti, A.M.; Harley, R.M.; Queiroz, L.P.; Wanderley, M.G.L. \& Pirani, J.R. 2000. Caracterização e endemismos nos campos rupestres da Cadeia do Espinhaço. Pp. 311-318. In: T.B. Cavalcanti \& B.M.T. Walter (eds.). Tópicos Atuais de Botânica. Brasília, EMBRAPA Recursos Genéticos.

Harley, R.M. \& Simmons, N.A. 1986. Florula of Mucugê: a descriptive check-list of a campo rupestre area. Kew, Royal Botanic Gardens.

Köppen, W.P. 1948. Climatologia: con un estudio de los climas de la tierra. México, Fondo de Cultura Económica.
Kronka, F.J.N.; Nalon, M.A.; Matsukuma, C.K.; Pavão, M.; Guillaumon, J.R.; Cavalli, A.C.; Giannotti, E.; Iwane, M.S.S.; Lima, L.M.P.R.; Montes, J.; Del Cali, I.H. \& Haack, P.G. 1998. Áreas do domínio do cerrado no Estado de São Paulo. São Paulo, Secretaria de Estado do Meio Ambiente, Instituto Florestal.

Machado Filho, L.; Ribeiro, M.W.; Gonzalez, S.R.; Schenini, C.A.; Santos Neto, A.; Palmeira, R.C.B.; Pires, J.L.; Teixeira, W. \& Castro, H.E.F. 1983. Geologia. Pp. 27-304. In: Projeto RadamBrasil. Levantamento de Recursos Naturais. v. 32. Rio de Janeiro, Ministério de Energia e Minas, Secretaria Geral.

Moreira, A.A.N. 1977. Relevo. Pp. 1-34. In: M.G. Galvão (coord.). Geografia do Brasil, Região Centro-Oeste. Rio de Janeiro, Fundação Instituto Brasileiro de Geografia e Estatística.

Moreira, A.A.N. \& Camelier, C. 1977. Relevo pp. 1-50. In: M.G. Galvão (coord.). Geografia do Brasil, Região Sudeste. Rio de Janeiro, Fundação Instituto Brasileiro de Geografia e Estatística.

Nakajima, J.N. \& Semir, J. 2001. Asteraceae do Parque Nacional da Serra da Canastra, Minas Gerais, Brasil. Revista Brasileira de Botânica 24: 471-478.

Nimer, E. 1989. Climatologia do Brasil. Rio de Janeiro, Instituto Brasileiro de Geografia e Estatística.

Ratter, J.A. \& Dargie, T.C.D. 1992. An analisys of the floristic composition of 26 cerrado areas in Brazil. Edinburgh Journal of Botany 49: 235-250.

Ratter, J.A.; Bridgewater, S.; Atkinson, R. \& Ribeiro, J.F. 1996. Analysis of the floristic composition of the Brazilian cerrado vegetation II: comparison of the wood vegetation of 98 areas. Edinburgh Journal of Botany 53: 153-180.

Ratter, J.A.; Bridgewater, S. \& Ribeiro, J.F. 2003. Analysis of the floristic composition of the Brazilian cerrado vegetation III: comparison of the wood vegetation of 376 areas. Edinburgh Journal of Botany 60: 57-109.

Ribeiro, J.F. \& Walter, M.B.T. 1998. Fitofisionomias do bioma Cerrado. Pp. 89-166. In: S.M. Sano \& S.P. Almeida (eds.). Cerrado: ambiente e flora. Planaltina, Embrapa.

Romero, R. \& Martins, A. 2002. Melastomataceae do Parque Nacional da Serra da Canastra, Minas Gerais, Brasil. Revista Brasileira de Botânica 25: 19-24.

Romero, R. 2002. Diversidade da flora dos campos rupestres de Goiás, sudoeste e sul de Minas Gerais. Pp. 81-86. In: E.L. Araújo; A.N. Moura; E.V.S.B. Sampaio; L.M.S. Gestinari \& J.M.T. Carneiro (eds.). Biodiversidade, Conservação e Uso Sustentável da Flora do Brasil. Recife, Universidade Federal Rural de Pernambuco, Sociedade Botânica do Brasil.

Ross, J.L.S. \& Moroz, I.C. 1997. Mapa geomorfológico do Estado de São Paulo - v. 1. Escala 1:500.000. São Paulo, Instituto de Pesquisa Tecnológicas, Fapesp.

Vitta, F.A. 2002. Diversidade e conservação da flora nos campos rupestres da Cadeia do Espinhaço em Minas Gerais. Pp. 90-94. In: E.L. Araújo; A.N. Moura; E.V.S.B. Sampaio; L.M.S. Gestinari \& J.M.T. Carneiro (eds.). Biodiversidade, Conservação e Uso Sustentável da Flora do Brasil. Recife, Universidade Federal Rural de Pernambuco, Sociedade Botânica do Brasil. 